\title{
The YAP/HIF-1a/miR-182/EGR2 axis is implicated in asthma severity through the control of Th17 cell differentiation
}

Jing Zhou' ${ }^{1}$ Ning Zhang ${ }^{2}$, Wei Zhang ${ }^{1}$, Caiju Lu ${ }^{1}$ and Fei $X u^{1 *}$

\begin{abstract}
Background: Asthma is a heterogeneous chronic inflammatory disease of the airway, involving reversible airflow limitation and airway remodeling. Thelper 17 (Th17) cells play an important role in the pathogenesis of allergic asthma. However, there is limited understanding of the signaling pathways controlling Th17 cell differentiation in asthma. The aim of this study was to investigate if the Yes-associated protein (YAP)/hypoxia inducible factor-1a (HIF-1a)/microRNA-182 (miR-182)/early growth response 2 (EGR2) axis is involved in mediating Th17 cell differentiation and disease severity in asthma.

Methods: The study included 29 pediatric patients with asthma, 22 healthy volunteers, ovalbumin-induced murine asthma models, and mouse naive CD4 ${ }^{+} \mathrm{T}$ cells. The subpopulation of Th17 cells was examined by flow cytometry. The levels of interleukin-17A were determined by enzyme linked immunosorbent assay. Chromatin immunoprecipitationquantitative polymerase chain reaction assays and dual-luciferase reporter gene assays were performed to examine interactions between HIF-1 $a$ and miR-182, and between miR-182 and EGR2.
\end{abstract}

Results: YAP, HIF-1a, and miR-182 were upregulated but EGR2 was downregulated in human and mouse peripheral blood mononuclear cells from the asthma group. Abundant expression of YAP and HIF-1a promoted miR-182 expression and then inhibited EGR2, a target of miR-182, thus enhancing Th17 differentiation and deteriorating asthma and lipid metabolism dysfunction. In addition, in vivo overexpression of EGR2 countered the promoting effect of the YAP/ HIF-1a/miR-182 axis on asthma and lipid metabolism dysfunction.

Conclusion: These results indicate that activation of the YAP/HIF-1a/miR-182/EGR2 axis may promote Th17 cell differentiation, exacerbate asthma development, and aggravate lipid metabolism dysfunction, thus suggesting a potential therapeutic target for asthma.

Keywords: YAP, HIF-1a, MiR-182, EGR2, Th17 cells, Differentiation, Asthma, Dyslipidemia

\section{Background}

Asthma is a frequently occurring disease of the airways, affecting about 300 million people worldwide [1]. The incidence of asthma is higher in high-income countries,

\footnotetext{
*Correspondence: drFeiXuuue@163.com

${ }^{1}$ Department of Respiratory Medicine, The First Affiliated Hospital of Nanchang University, No. 17, Yongwai Street, Donghu District, Nanchang 330006, People's Republic of China

Full list of author information is available at the end of the article
}

whereas asthma-related mortality is predictably higher in lower-income countries [2]. Albeit the global prevalence of asthma is about $4-5 \%$, this figure can vary as much as 21-fold across different countries [2]. The well-recognized pathophysiology of asthma consists of recurrent lung epithelial inflammation, bronchial smooth muscle hyperreactivity, chronic lung tissue remodeling, and excessive mucus production, leading to reversible airflow restriction [3]. The vast majority of patients with asthma also present with concomitant 
rhinitis, which can further amplify the risk for developing asthma with heightened bronchial hyperresponsiveness and reactivity to a variety of stimuli [4]. The current management of asthma primarily focuses on alleviating disease severity and choosing the appropriate medical therapy to control symptoms and reduce the risk of exacerbations; however, some patients still experience acute exacerbation of symptoms and a loss of disease control $[5,6]$, highlighting an urgent need to fully elucidate the underlying pathogenesis and develop more efficacious treatment regimens to better tackle asthma [7].

Recent data demonstrated that Yes-associated protein (YAP), a downstream target of the Hippo pathway, is implicated in the generation and maintenance of cancer-associated fibroblasts and vascular smooth muscle cell differentiation $[8,9]$. YAP holds significant potential as an anticancer immunotherapeutic target owing to the fact that YAP deficiency induces dysfunctional Tregs unable to suppress anti-tumor immunity or promote tumor growth [10]. Silencing YAP has been shown to facilitate airway smooth muscle cell proliferation, migration, and contraction induced by sphingosine-1-phosphate in asthma [11]. In addition, YAP can bind to hypoxia-inducible factor $1 \alpha$ (HIF-1 $\alpha)$ and maintain its protein stability, thus promoting hepatocellular carcinoma cell glycolysis under hypoxic stress [12]. During the induction phase of asthma, treatment with HIF-1 $\alpha$ inhibitor is shown to decrease eosinophilia in bronchoalveolar lavage samples, lung parenchyma, and total lung inflammation [13]. Moreover, HIF-1 $\alpha$ has also been shown to promote the expression of microRNA-182 (miR-182) [14]. This is notable as miR-182 is also documented as upregulated during T-helper 17 (Th17) cell differentiation [15]. Accruing research has revealed a significant correlation of activated Th17 cells with the progression of asthma [16-18]. Moreover, early growth response 2 (EGR2), known as a transcription factor negatively regulating T-cell activation [19], has been reported to reduce Th17 cell differentiation [20]. In another instance, a reduction of high-density lipoprotein cholesterol (HDL-C) has been linked to increased numbers of Th17 cells [21]. In a related finding, decreased HDL-C levels have been found in children with asthma [22]. Therefore, we sought to determine if YAP and HIF- $1 \alpha$ function through a pathway that involves low HDL-C-induced increased Th17 cells in the pathogenesis of pediatric asthma. These results could potentially reveal a hitherto undocumented link between HIF-1 $\alpha$, miR-182, EGR2, and Th17 cells in context of asthma and therefore, we investigated if miR-182 and EGR2 were involved in asthma pathogenesis.

\section{Results}

YAP, HIF-1 $a$ and miR-182 are upregulated and HDL-C levels are decreased in asthma

With an aim to identify the putative genes implicated in the development of asthma, a differential gene expression analysis was performed on the asthma-related GSE27876 dataset retrieved from the Gene Expression Omnibus database (https://www.ncbi.nlm.nih.gov/gds) using the R package Limma (http://www.bioconductor.org/packa ges/release/bioc/html/limma.html). The GSE27876 dataset (comparing peripheral blood cells from mild and severe asthma that were selected from patients classified into the asthma treatment step 4, in accordance with the criteria described in the Global Initiative for Asthma) was identified. It comprised of 5 normal samples and 10 asthma samples where peripheral blood was the sample type. With the selected cutoff criteria (|logFoldChange $\mid>1.5$, and $p<0.05$ ), a total of 331 differentially expressed genes (DEGs) were identified (Additional file 1: Fig. S1A, Additional file 9: Table S1). In addition, 318 human transcription factors were identified from the Cistrome database (http://cistrome.org/). A Venn diagram analysis of the 331 DEGs and 318 human transcription factors revealed 5 transcription factors were found at the intersection, namely H2AFX, NOTCH1, RUNX1T1, USF1, and YAP1 (Additional file 1: Fig. S1B, Additional file 10: Table S2). Subsequently, protein-protein interactions (PPI) between these aforementioned 5 transcription factors and their related genes were identified using the GeneMANIA web-based tool (http://genemania.org/). As illustrated in Additional file 1: Fig. S1C and Table 1, the 2 most robustly related transcription factors were identified as NOTCH1 and YAP1. YAP1 has been widely correlated with asthma [23, 24], and also known to influence disease progression by mediating HIF- $1 \alpha$ in liver cancer and pancreatic cancer [12, 25]. As demonstrated previously, NOTCH1 is involved in the promotion of the GATA3-mediated Th2 response (immunity), which makes NOTCH1 an unlikely candidate responsible for the non-Th2 inflammatory pattern in asthma [26]. As a result, YAP1 (YAP) was selected as the upstream gene for investigation in the current study. In addition, using the Multi Experiment Matrix (MEM) web based tool (https://biit.cs.ut.ee/mem/index.cgi) it was predicted that YAP1 was significantly co-expressed with HIF1A (Additional file 1: Fig. S1D). Using the hTFtarget tool (http:// bioinfo.life.hust.edu.cn/hTFtarget\#!/) YAP1 was found to target the HIF1A gene (Additional file 1: Fig. S1E). Based on these aforementioned in silico analyses and previous experimental data, we hypothesized that YAP1 (YAP) may affect the development of asthma through regulation of HIF1A (HIF-1 $\alpha$ ), and consequently designed the current study in order to validate this assumption. 
Table 1 Sequences for mimic and inhibitor

\begin{tabular}{|c|c|}
\hline Target gene & Sequence $\left(5^{\prime}-3^{\prime}\right)$ \\
\hline sh-YAP-1 & AGTGCAGCAGAATATGATG \\
\hline sh-YAP-2 & GAGATGGATACAGGTGATA \\
\hline sh-HIF-a-1 & $\begin{array}{l}\text { F: GATCCCCATCCAGAAGTCACTGGAACTTTCAAGAGAAGTTCCAGTGACTCTGGATTTTTGGAAA } \\
\text { R: TCGAAAAGGTTTTTTTAGGTCTCAGTGACCTTGAAGAGAACTTTCAAGGTCACTGAGACCTACCC }\end{array}$ \\
\hline sh-HIF-1a-2 & $\begin{array}{l}\text { F: AATTGATGGAACATGATGGTTCACTTCAAGAGAGTGAACCATCATGTTCCATTTTTT } \\
\text { R: CTAGAAAAAAATGGAACATGATGGTTCACTCTCTTGAAGTGAACCATCATGTTCCATC }\end{array}$ \\
\hline miR-182 inhibitor & TTCTACCATTGCCAA' \\
\hline sh-EGR2-1 & $\begin{array}{l}\text { F: GATCCATGCGTAACTTCAGTCGTAAGAGAACTTTACGACTGAAGTTACGCATTTTTTTCTCGAGG } \\
\text { R: AATTCCTCGAGAAAAAAATGCGTAACTTCAGTCGTAAAGTTCTCTTACGACTGAAGTTACGCATG }\end{array}$ \\
\hline miR-182 mimic & $\begin{array}{l}\text { F: UUUGGCAAUGGUAGAACUCACACU } \\
\text { R: UGUGAGUUCUACCAUUGCCAAAUU }\end{array}$ \\
\hline
\end{tabular}

YAP Yes-associated protein, HIF-1a hypoxia-inducible factor $1 a$, miR microRNA, EGR2 early growth response $2, F$ forward, $R$ reverse

The results of hematoxylin-eosin staining illustrated that the ovalbumin (OVA)-induced asthmatic mice presented with airway remodeling $(p<0.05$; Additional file 2: Fig. S2A) and increased thickening of the airway smooth muscles, airway wall, and airway epithelium mucosa relative to the sham-operated mice $(p<0.05$; Additional file 2 : Fig. S2B). These findings verified the successful establishment of OVA-induced murine asthma models. Next, the proportion of Th17 cells was assessed in human peripheral blood mononuclear cells (PBMCs) and mouse spleen cells using flow cytometry, which revealed an increased proportion of Th17 cells in both patients and mice with asthma $(p<0.05$; Fig. 1a). Furthermore, enzyme linked immunosorbent assay (ELISA) displayed that the serum levels of interleukin-17A (IL-17A) were up-regulated in patients with asthma $(p<0.05$; Fig. $1 \mathrm{~b})$. In addition, reverse transcription quantitative polymerase reaction (RT-qPCR) and Western blot analysis determined that retineic-acid-receptor-related orphan nuclear receptor gamma (ROR $\gamma \mathrm{t}$ ) mRNA and protein expression were elevated in PBMCs of patients with asthma $(p<0.05$; Fig. 1c, d).

Using RT-qPCR and Western blot analysis YAP and HIF-1 $\alpha$ expression levels were found significantly higher in patients with asthma $(p<0.05$; Fig. $1 \mathrm{e}, \mathrm{f})$. As a significant association between asthma and the serum HDL-C has been described [22], HDL-C was employed as a marker to assess asthma disease severity in the current study and as depicted in Fig. 1g, serum HDL-C levels were significantly elevated in patients with asthma $(p<0.05)$. Evidence further suggests that HIF-1 $\alpha$ can promote the expression of miR-182 [14], whereas the miR-183/96/182 cluster is known to be significantly upregulated in Th17 differentiation [15]. Therefore, we speculated that the HIF- $1 \alpha$ signaling pathway might promote Th17 differentiation by regulating miR-182. Therefore, RT-qPCR quantification of miR-96/182/183 expression levels in human
PBMCs was performed, showing an upward trend in the miR-96/182/183 expression in patients with asthma, with a particularly pronounced increase in miR-182 expression $(p<0.05$; Fig. 1h). Largely in agreement with the results observed in human PBMCs, in mice with asthma, serum IL-17A content was significantly increased (Additional file 3: Fig. S3A, $p<0.05)$, ROR $\gamma$ t was upregulated in spleen cells (Additional file 3: Fig. S3B, C, $p<0.05$ ) accompanied by higher levels of YAP/HIF-1 $\alpha$ (Additional file 3: Fig. S3D, E, $p<0.05$ ), and lower HDL-C level (Additional file 3: Fig. S3F, $p<0.05)$ along with upregulation of miR-96/182/183 $(p<0.05)$, among which the increase in miR-182 expression was pronounced (Additional file 3: Fig. S3G, $p<0.05)$. Collectively, these results suggested that miR-182, YAP and HIF- $1 \alpha$ were upregulated, and serum HDL-C level was decreased in asthma.

\section{YAP and HIF-1a promote the differentiation of $\mathrm{CD}^{+}{ }^{+} \mathrm{T}$ Cells into Th17 cells}

In order to further verify whether YAP and HIF-1 $\alpha$ were also upregulated in Th17 cells, we first induced the differentiation of $\mathrm{CD}^{+} \mathrm{T}$ cells into Th17 cells in vitro. Next, RT-qPCR and Western blot analysis were applied to determine the expression levels of YAP and HIF-1 $\alpha$ in the differentiated TH17 cells. As illustrated in Fig. 2a, b, the mRNA and protein expression of YAP and HIF- $1 \alpha$ were increased in Th17 cells compared to $\mathrm{CD}^{+} \mathrm{T}$ cells $(p<0.05)$.

Additionally, the infection efficiency of YAP/HIF- $1 \alpha$ overexpression or knockdown in the TH17 cells was evaluated and confirmed by means of RT-qPCR and Western blot analysis (Additional file 4: Fig. S4A, B), where sh-YAP-1 and sh-HIF- $1 \alpha-1$ exhibited superior efficiency, and were thus used for further experimentation $(p<0.05)$. Western blot analysis demonstrated that the protein expression of HIF- $1 \alpha$ was increased in cells with 


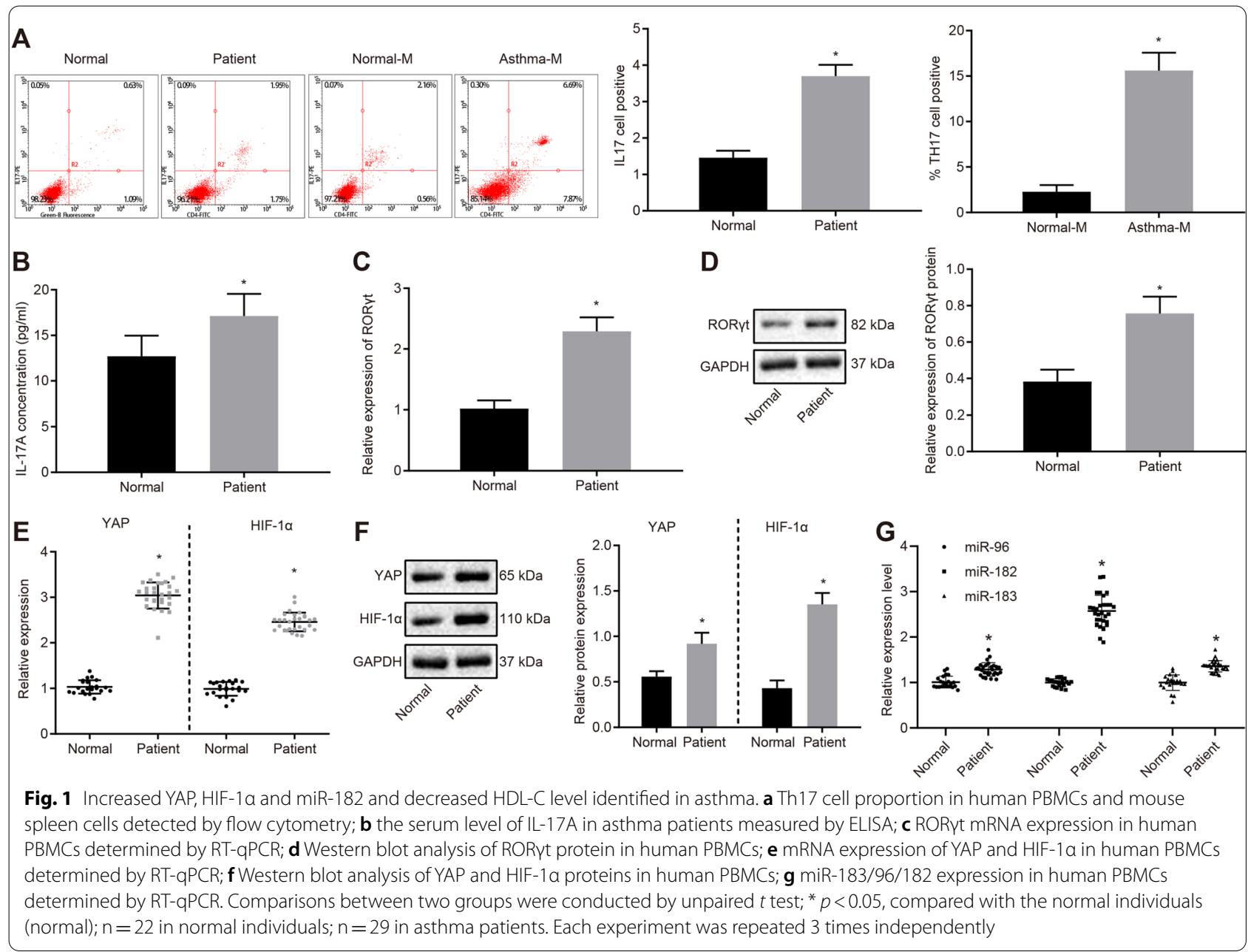

YAP overexpression, but decreased in YAP knockdown cells ( $p<0.05$; Additional file 4: Fig. S4C).

To further investigate whether YAP/HIF- $1 \alpha$ promoted the differentiation of $\mathrm{CD} 4^{+} \mathrm{T}$ cells into Th17 cells, Western blot analysis was used to quantify protein expression levels of YAP and HIF- $1 \alpha$ in Th17 cells subjected to different treatments. As shown in Fig. 2c, cells treated with overexpressed YAP or with a combination of YAP overexpression and HIF- $1 \alpha$ silencing exhibited significantly increased YAP protein expression $(p<0.05)$. However, no significant changes were noted in the YAP protein expression levels between HIF- $1 \alpha$ overexpressing cells and HIF- $1 \alpha$ silenced cells $(p>0.05)$. When YAP or HIF- $1 \alpha$ were overexpressed, HIF-1 $\alpha$ expression was increased in Th17 cells $(p<0.05)$. The opposite trends were observed in Th17 cells upon YAP knockdown or/and HIF-1 $\alpha$ knockdown treatment $(p<0.05)$.

Thereafter, the Th17 cell proportion was measured using flow cytometry (Fig. 2d), showing that YAP or HIF-1 $\alpha$ overexpression led to higher Th17 cell proportion, while YAP or HIF- $1 \alpha$ silencing resulted in reduced Th17 cell proportion $(p<0.05)$. In addition, increase in Th17 cell proportion caused by YAP overexpression was inhibited by HIF- $1 \alpha$ silencing $(p<0.05)$.

The ELISA assays (Fig. 2e), RT-qPCR and Western blot analysis (Fig. 2f, g) revealed that IL-17A levels and RORyt expression were both elevated as a result of YAP or HIF- $1 \alpha$ overexpression, but reduced in the absence of YAP or HIF- $1 \alpha \quad(p<0.05)$. Additionally, increased IL-17A levels and ROR $\gamma \mathrm{t}$ expression owing to YAP overexpression were found to be inhibited by HIF-1 $\alpha$ silencing $(p<0.05)$.

These findings demonstrated that the differentiation of $\mathrm{CD} 4^{+} \mathrm{T}$ cells into Th17 cells was promoted by overexpression of YAP and HIF- $1 \alpha$.

\section{HIF-1a promotes the differentiation of $\mathrm{CD}^{+} \mathrm{T}$ Cells into Th 17 cells by upregulating miR-182}

In the following experiments, we aimed to uncover the mechanism of HIF- $1 \alpha$ involvement in Th17 cell differentiation. RT-qPCR assay showed that miR-182 


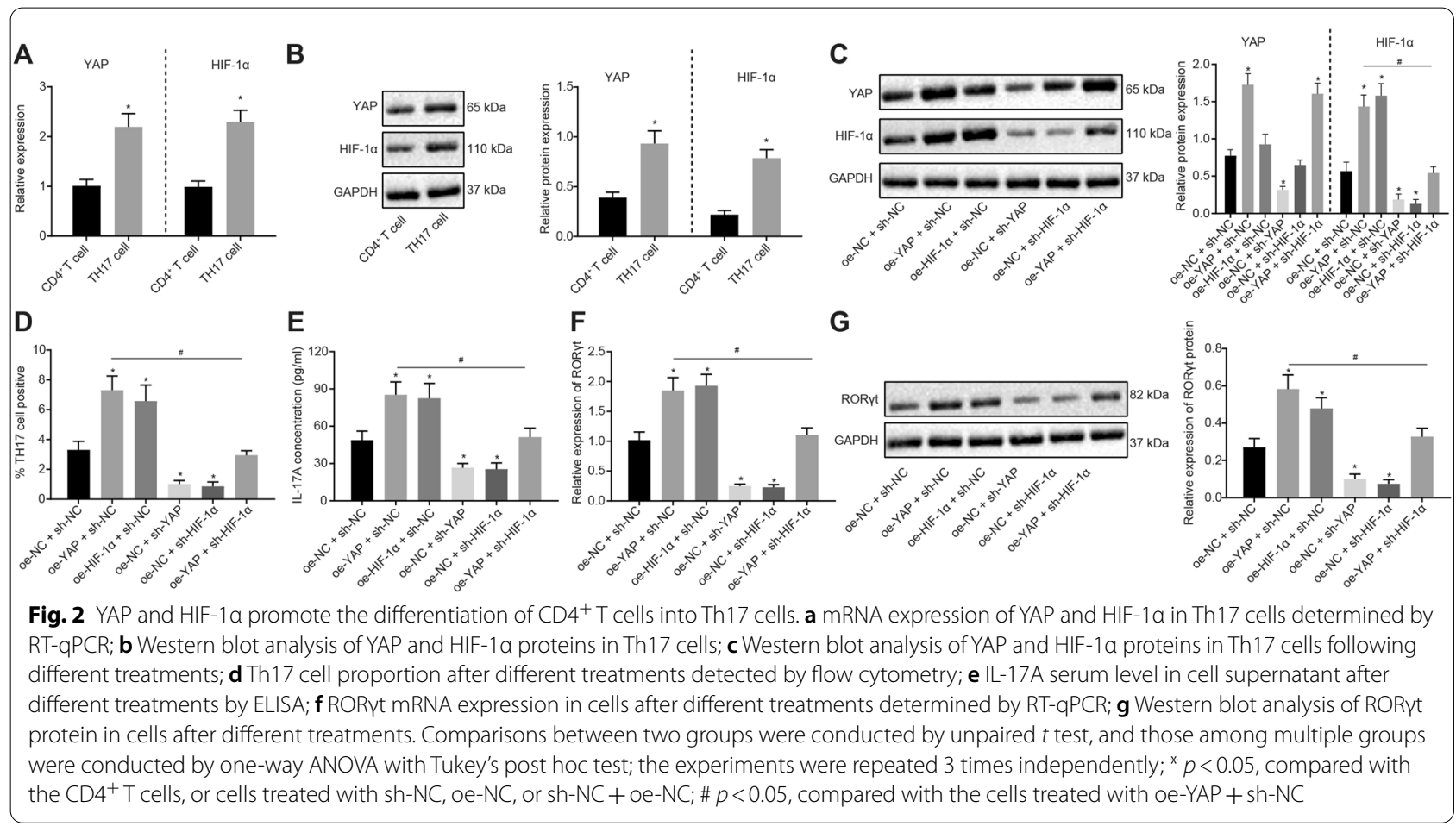

expression was upregulated in Th17 cells relative to that in $\mathrm{CD} 4^{+} \mathrm{T}$ cells $(p<0.05$; Fig. $3 \mathrm{a})$. In addition, Western blot analysis demonstrated that the protein expression of HIF- $1 \alpha$ was elevated upon treatment with HIF-1 $\alpha$ overexpression in HEK293T cells with miR182-wild type (wt) or miR-182-mutant (mut) $(p<0.05)$. A dual luciferase reporter gene assay revealed that HIF-1 $\alpha$ overexpression led to a significant increase in the luciferase activity of miR-182-wt in HEK293T cells $(p<0.05$; Fig. $3 \mathrm{~b})$. Moreover, the chromatin immunoprecipitation (ChIP) assay illustrated that HIF-1 $\alpha$ could bind to the miR-182 promoter (Fig. 3c), and compared to the immunoglobulin G (IgG) control, the enrichment of HIF- $1 \alpha$ in the miR-182 promoter was notably increased, and was further promoted by HIF- $1 \alpha$ overexpression $(p<0.05)$. In support, RT-qPCR assays demonstrated that the expression of miR-182 was increased in cells treated with oe-HIF- $1 \alpha$ or oe-YAP, but was decreased in cells treated with sh-HIF-1 $\alpha$ or sh-YAP $(p<0.05$; Fig. $3 \mathrm{~d})$. These results indicated that HIF- $1 \alpha$ promoted the expression of miR-182 in HEK293T cells.

Next, we examined whether YAP/HIF- $1 \alpha$ promoted the differentiation of $\mathrm{CD}^{+} \mathrm{T}$ cells into Th17 cells. RT-qPCR assays showed reduced miR-182 expression in cells treated with oe-NC+miR-182 inhibitor, oe-HIF- $1 \alpha+$ miR-182 inhibitor or oe-YAP + miR-182 inhibitor, and elevated miR-182 levels in cells treated with sh-NC+miR-182 mimic, sh-HIF- $1 \alpha+$ miR-182 mimic or sh-YAP + miR-182 mimic $(p<0.05)$. RTqPCR and Western blot analysis also revealed increased expression of HIF-1 $\alpha$ in Th17 cells treated with oe-HIF- $1 \alpha+$ miR-182 inhibitor, oe-HIF- $1 \alpha+$ inhibitor$\mathrm{NC}$, oe-YAP + miR-182 inhibitor or oe-YAP + inhibitor-NC, with decreased levels in Th17 cells treated with sh-HIF-1 $\alpha+$ miR-182 mimic, sh-NC + miR-182 mimic, sh-YAP + miR-182 mimic or sh-NC + miR-182 mimic $(p<0.05$; Additional file 2 Fig. S2A, B).

The results of flow cytometry further displayed that Th17 cell proportion was reduced in response to treatment with oe-NC+miR-182 inhibitor, oe-HIF- $1 \alpha+$ miR-182 inhibitor, oe-YAP + miR-182 inhibitor, sh-HIF- $1 \alpha+$ mimic-NC or sh-YAP + mimic$\mathrm{NC}$, but opposite trends were noted upon treatment with sh-NC + miR-182 mimic, oe-HIF- $1 \alpha+$ inhibitor-NC, oeYAP + inhibitor-NC, sh-HIF- $1 \alpha+$ miR-182 mimic or shYAP + miR-182 mimic $(p<0.05$; Fig. 3e).

Further, ELISA assays demonstrated that IL-17A levels in the supernatant were decreased upon treatment 


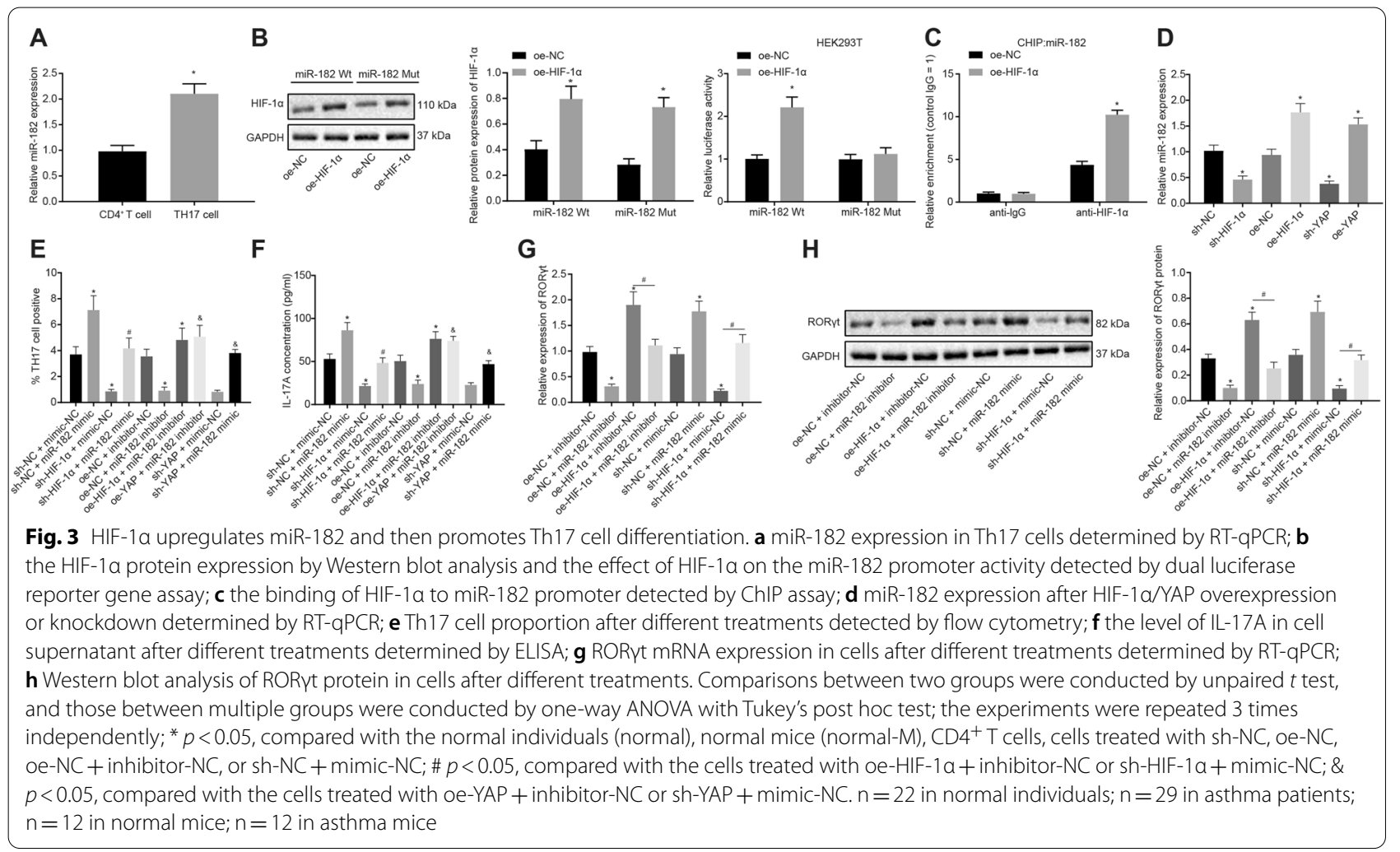

with oe-NC + miR-182 inhibitor, which was reversed by sh-NC + miR-182 mimic, oe-HIF- $1 \alpha+$ inhibitor-NC or oe-YAP + inhibitor-NC treatment. In addition, IL-17A levels were found to be diminished in the presence of oe-HIF- $1 \alpha+$ miR-182 inhibitor or oe-YAP + miR-182 inhibitor. Silencing HIF-1 $\alpha$ or YAP led to decreased IL17 A levels, while treatment with sh-HIF- $1 \alpha+$ miR-182 mimic or sh-YAP + miR-182 mimic abrogated the trend (all $p<0.05$; Fig. 3f).

RT-qPCR and Western blot analysis also illustrated that ROR $\gamma$ t expression was diminished in cells upon miR-182 downregulation or HIF- $1 \alpha$ downregulation, but elevated in cells treated with miR-182 upregulation or HIF- $1 \alpha$ upregulation. Meanwhile, dual treatment with oe-HIF-1 $\alpha$ and miR-182 inhibitor inhibited the ROR $\gamma$ t expression, while treatment with both sh-HIF- $1 \alpha$ and miR-182 mimic resulted in increased RORyt levels ( $p<0.05$; Fig. 3g, h).

These findings revealed that HIF- $1 \alpha$ upregulated miR182 expression and consequently promoted differentiation of $\mathrm{CD}^{+}{ }^{+} \mathrm{T}$ cells into Th17 cells.

\section{EGR2 is downregulated in asthma}

At the cutoff criteria ( $|\operatorname{logFoldChange~}|>1$, and $p<0.05$ ), 27 DEGs were obtained from the GSE64913 dataset (epithelial cells from central airways and from peripheral airways), which included 42 normal samples and 28 asthma samples (Fig. 4a, Additional file 11: Table S3). Next, 18,165 downstream genes of miR-182 were identified from the mirDIP database (score class: medium; http://ophid.utoronto.ca/mirDIP/) and their human transcription factors in the Cistrome database were identified by means of a Venn diagram. EGR2 was found to be a key downstream transcription factor for miR-182 (Fig. 4b, Additional file 12: Table S4). EGR2 has also been reported to exert an inhibitory role on Th17 cell differentiation [21], and therefore, we evaluated its expression pattern in human PBMCs (Fig. 4c, d) and mouse spleen cells (Fig. 4e, f) using RT-qPCR and Western blot assays. The results demonstrated that EGR2 expression was downregulated in asthma $(p<0.05)$. As a result, we postulated that miR-182 might promote Th17 cell differentiation via inhibition of EGR2.

\section{miR-182 promotes the differentiation of $\mathrm{CD}^{+} \mathrm{T}$ Cells into Th17 Cells by inhibiting EGR2}

Next, we aimed to elucidate and verify the relationship between miR-182 and EGR2. The online resource (http:// starbase.sysu.edu.cn/) was used to predict binding site presence between miR-182 and EGR2 3'untranslated region (3'UTR) (Fig. 5a). RT-qPCR analysis revealed that 
miR-182 expression was increased in HEK293T cells cotransfected with miR-182 mimic and EGR2-wt or EGR2mut $(p<0.05)$. Thereafter, a dual luciferase reporter gene assay demonstrated that the luciferase activity of EGR2-wt was reduced $(p<0.05)$, while that of EGR2-mut showed no changes in HEK293T cells following miR182 mimic transfection ( $p>0.05$; Fig. $5 b)$. The results of RT-qPCR and Western blot analysis shown in Fig. 5c, d indicated a diminished expression of EGR2 in miR-182 mimic-treated cells and elevated expression of EGR2 in miR-182 inhibitor-treated cells $(p<0.05)$. These results reflected that miR-182 targeted EGR2 and inhibited its expression.

Going further, we examined whether miR-182 could induce Th17 cell differentiation via EGR2 inhibition. RT-qPCR and Western blot analysis showed that EGR2 expression was increased upon EGR2 overexpression but decreased following sh-EGR2-1 and sh-EGR2-2 treatment $(p<0.05$; Additional file 6: Fig. S6A, B). Due to the higher silencing efficiency of EGR2-1 compared to EGR2-2, sh-EGR2-1 was chosen for subsequent experimentation.

Using RT-qPCR and Western blot analysis (Additional file 6: Fig. S6C, D), we showed that cells treated with oeEGR2 or both miR-182 mimic and oe-EGR2 displayed elevated expression of EGR2, while those treated with sh-EGR2 exhibited reduced EGR2 $(p<0.05)$. Moreover, cells treated with miR-182 mimic showed increased miR182 expression and decreased EGR2 expression $(p<0.05)$. However, cells treated with miR-182 mimic did not markedly differ in miR-182 expression from cells treated with both miR-182 mimic and oe-EGR2 $(p>0.05)$. In addition, cells treated with miR-182 inhibitor exhibited reduced miR-182 expression and elevated EGR2 expression $(p<0.05)$. However, as compared to the cells treated with miR-182 inhibitor, miR-182 expression was not significantly different from the cells co-treated with miR182 inhibitor and sh-EGR2 $(p>0.05)$, while EGR2 was decreased $(p<0.05)$.

Flow cytometry was applied to assess the Th17 cell proportion in treated cells. As illustrated in Fig. 5e, EGR2 overexpression or miR-182 inhibitor treatment led to reduced Th17 cell proportion, while EGR2 knockdown or miR-182 mimic enhanced the Th17 cell proportion $(p<0.05)$. When compared with miR-182 mimic treatment alone, the combination treatment of miR-182 mimic and EGR2 overexpression resulted in a significantly decreased Th17 cell proportion $(p<0.05)$. At the same time, when compared with miR-182 inhibitor treatment alone, the combination treatment of miR-182 inhibitor and EGR2 silencing resulted in increased Th17 cell proportion $(p<0.05)$.
ELISA was performed to quantify IL-17A levels $(p<0.05$; Fig. 5f), while RT-qPCR and Western blot analysis were conducted to determine the ROR $\gamma t$ expression patterns $(p<0.05$; Fig. 5 g, h). IL-17A levels and ROR $\gamma t$ expression were both decreased in cells with miR-182 downregulation or EGR2 upregulation, and elevated in cells with miR-182 upregulation or EGR2 downregulation $(p<0.05)$. When compared with miR-182 mimic treatment, the combination treatment of miR-182 mimic and EGR2 overexpression led to reduced IL-17A levels and RORyt expression $(p<0.05)$. When compared with miR-182 inhibitor treatment, the combination treatment of miR-182 inhibitor and EGR2 silencing exhibited elevated IL-17A levels and RORyt expression $(p<0.05)$.

Collectively, these findings suggested that miR-182 accelerated the differentiation of $\mathrm{CD} 4^{+} \mathrm{T}$ cells into Th17 cells by inhibiting the expression of EGR2.

\section{EGR2 inhibits the Th17 cell differentiation induced by YAP/ HIF-1a/miR-182}

The aforementioned findings suggested that EGR2 overexpression could inhibit miR-182-induced Th17 cell differentiation. In this section of experiments, we investigated whether EGR2 inhibited the Th17 cell differentiation evoked by the YAP/HIF- $1 \alpha$ axis. RT-qPCR and Western blot analysis (Additional file 7: Fig. S7A, B) displayed that the YAP expression was increased in $\mathrm{CD} 4^{+} \mathrm{T}$ cells upon YAP overexpression alone or both overexpression of YAP and EGR2 $(p<0.05)$. The HIF-1 $\alpha$ expression was also increased upon HIF-1 $\alpha$ overexpression alone or both overexpression of HIF-1 $\alpha$ and EGR2 $(p<0.05)$. In addition, cells with both overexpression of YAP and EGR2 exhibited higher EGR2 expression relative to those with YAP overexpression alone, and the cells with both overexpression of HIF-1 $\alpha$ and EGR2 also exhibited higher EGR2 expression compared to those with HIF-1 $\alpha$ overexpression alone $(p<0.05)$. Flow cytometry results (Fig. 6a) depicted that YAP or HIF-1 $\alpha$ overexpression promoted Th17 cell proportion, which was inhibited by EGR2 overexpression $(p<0.05)$.

Finally, Fig. $6 \mathrm{~b}$ illustrates the results of IL-17A levels measured by ELISA and Fig. 6c, d display the ROR $\gamma \mathrm{t}$ expression patterns determined by RT-qPCR and Western blot analysis. IL-17A levels and RORyt expression were both elevated in cells with YAP or HIF-1 $\alpha$ overexpression, while EGR2 overexpression led to a reversal of these trends $(p<0.05)$.

These data demonstrated that the differentiation of $\mathrm{CD} 4^{+} \mathrm{T}$ cells into Th17 cells induced by the YAP/HIF-1 $\alpha /$ miR-182 axis was inhibited by overexpression of EGR2. 


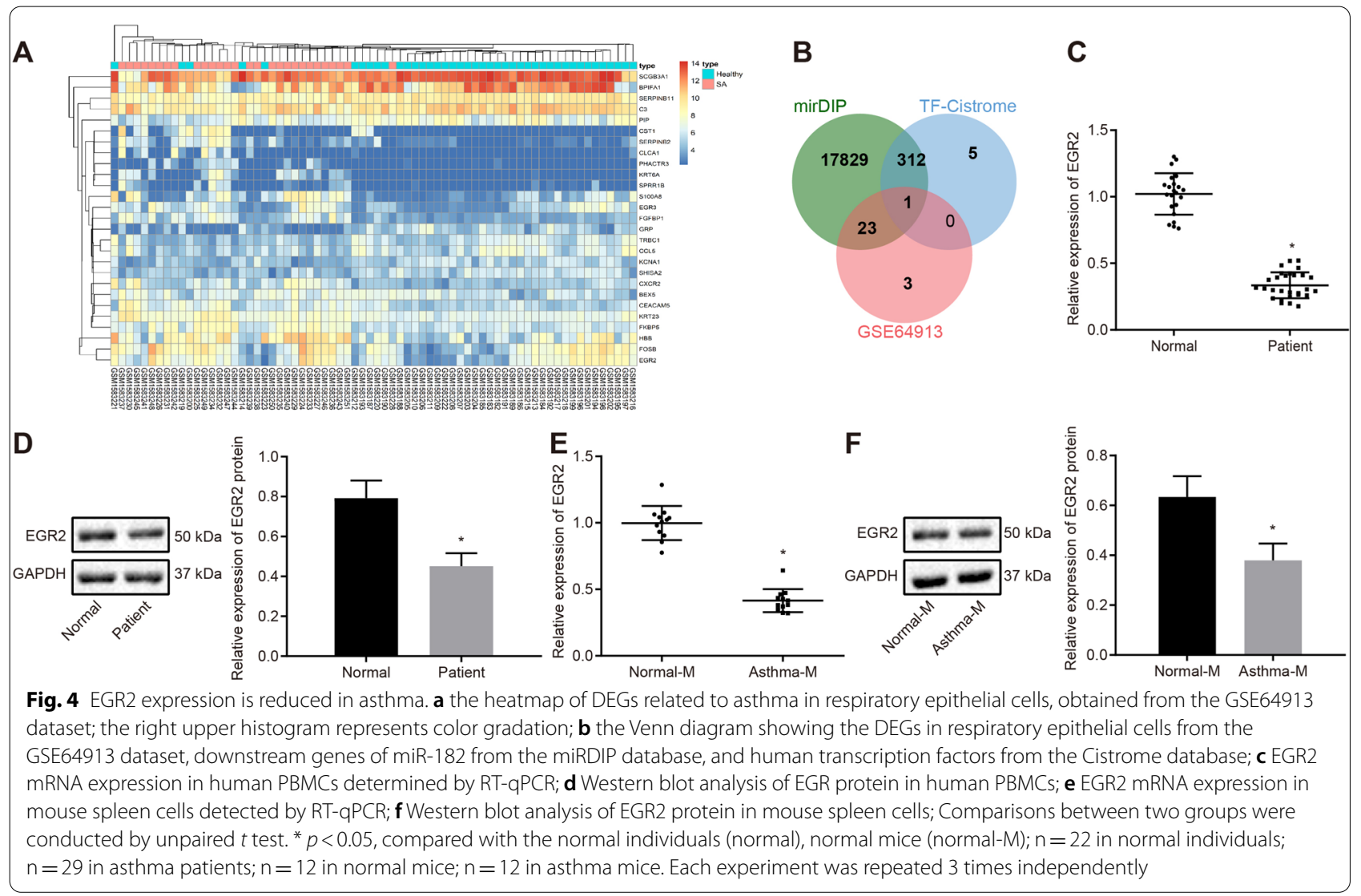

\section{Overexpression of EGR2 alleviates asthma and lipid metabolism dysfunction by inhibiting the YAP/HIF-1a/ miR-182 axis in vivo}

Next, we sought to verify the results in vivo. RT-qPCR was performed to determine the miR-182 expression patterns, and RT-qPCR and Western blot analysis were used to determine the protein expression patterns of YAP, HIF-1 $\alpha$, and EGR2 in mice (Additional file 8: Fig. S8A, B). Asthma mice presented with elevated YAP and HIF- $1 \alpha$ expression, but reduced EGR2 expression $(p<0.05)$. Mice with YAP overexpression treatment or dual treatment with YAP over-expression and EGR2 overexpression exhibited increased expression of YAP $(p<0.05)$. In addition, the treatments with YAP overexpression alone, HIF- $1 \alpha$ overexpression alone, both overexpression of YAP and EGR2, as well as both overexpression of HIF- $1 \alpha$ and EGR2 resulted in increased expression of miR-182 and HIF- $1 \alpha(p<0.05)$. However, YAP overexpression, HIF-1 $\alpha$ overexpression, or miR-182 overexpression resulted in decreased EGR2 expression, which could be rescued by EGR2 overexpression $(p<0.05)$. In addition, when compared with the mice treated with mimic$\mathrm{NC}$ and oe-NC, the expression level of miR-182 was significantly higher in the mice treated with both EGR2 overexpression and miR-182 mimic $(p<0.05)$.

Thereafter, the Th17 cell proportion in mouse spleen was assessed using flow cytometry. The results are displayed in Fig. 7a, showing an increased Th17 cell proportion in the asthma mice and the mice treated with overexpressed YAP, overexpressed HIF- $1 \alpha$, or overexpressed miR-182, while inhibited by overexpressed EGR2 $(p<0.05)$. In addition, ELISA assessment of IL-17A levels (Fig. 7b) and RT-qPCR and Western blot to determine the RORyt expression patterns (Fig. 7c, d) showed IL-17A levels and ROR $\gamma t$ expression were elevated in the asthma mice and the mice with overexpressed YAP, overexpressed HIF- $1 \alpha$, or overexpressed miR-182, which was inhibited by EGR2 overexpression $(p<0.05)$.

The Th17 cell proportion in mouse lung tissues evaluated using flow cytometry showed an increase in the asthma mice and the mice with overexpressed YAP, overexpressed HIF-1 $\alpha$, or overexpressed miR-182, which was, however, counteracted by the treatment with oe-YAP + oe-EGR2, oe-HIF- $1 \alpha+$ oe-EGR2 or miR182 mimic +oe-EGR2 $(p<0.05$; Fig. $7 \mathrm{e})$. Besides, as $\alpha$-SMA immunofluorescence staining results show, the 


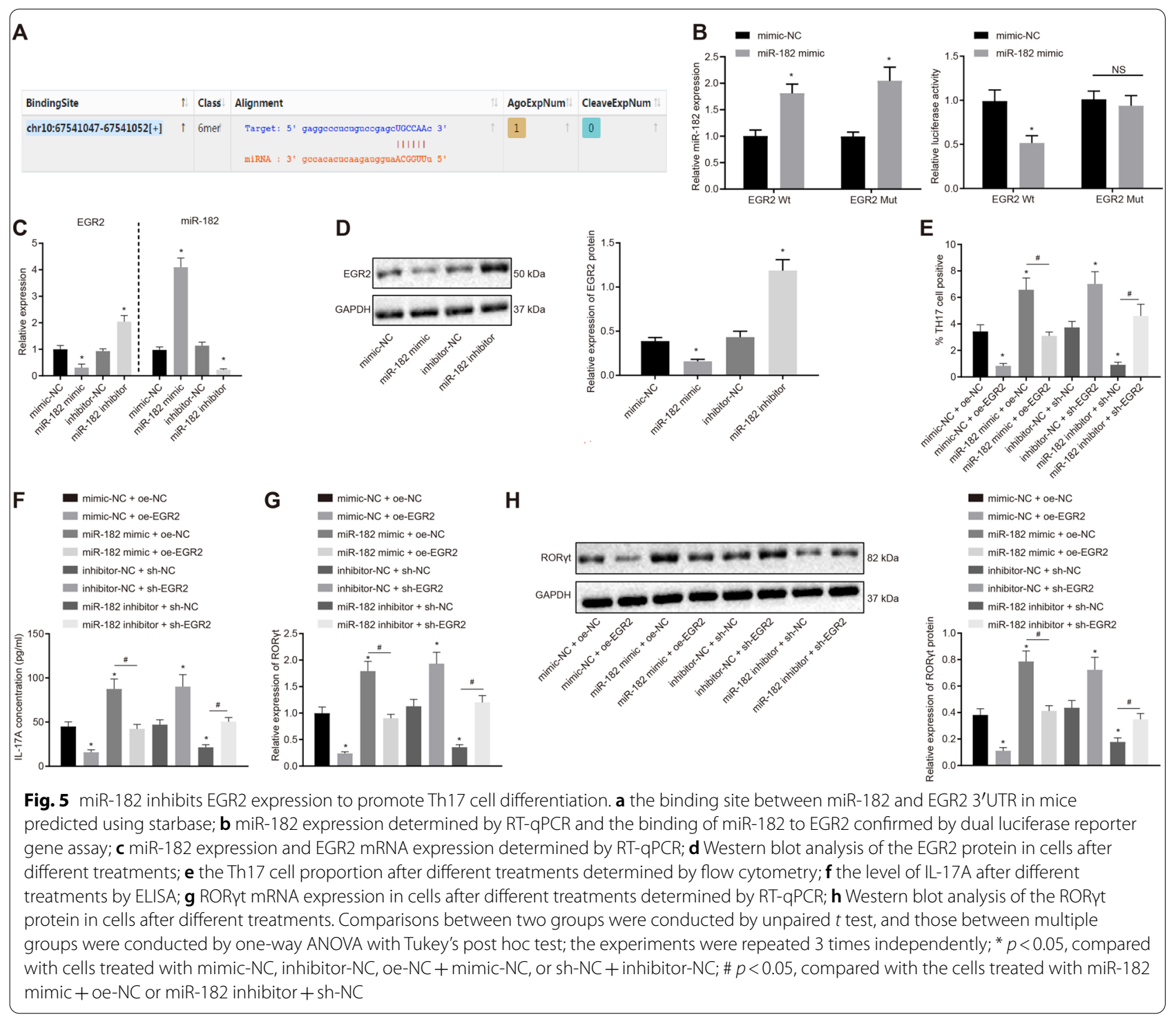

expression of $\alpha$-SMA was elevated in the lung tissues of asthma mice, which was also increased in the asthma mice treated with overexpressed YAP, overexpressed HIF-1 $\alpha$, or overexpressed miR-182, whereas EGR2 overexpression significantly inhibited the expression of $\alpha$-SMA (Fig. 7f). Furthermore, hematoxylin-eosin staining results demonstrated significant airway remodeling and increased thickening of the airway smooth muscles, airway wall, and airway epithelium mucosa in the asthma mice and mice with overexpressed YAP, overexpressed HIF- $1 \alpha$, or overexpressed miR-182, which was significantly inhibited by EGR2 overexpression $(p<0.05$; Fig. $7 \mathrm{~g}$ ).

HDL-C level was found decreased in serum from patients with asthma (Additional file 5: Fig S5A). In addition, asthma mice treated with oe-YAP, oe-HIF- $1 \alpha$, or
miR-182 mimic presented with reduced HDL-C levels, while increased HDL-C levels were found in response to EGR2 overexpression ( $p<0.05$; Additional file 5: Fig S5B).

These findings indicated that the overexpression of EGR2 alleviated the exacerbated asthma and lipid metabolism dysfunction evoked by YAP/HIF-1 $\alpha / m i R-182$ signaling in vivo.

\section{Discussion}

Asthma is a common disease affecting millions of people worldwide, characterized by upper airway inflammation and chronic nature [27]. In the current study, we aimed to investigate a potential molecular mechanism active in the development of asthma, and found that the YAP/HIF- $1 \alpha$ factors could potentially augment Th17 cell 
A
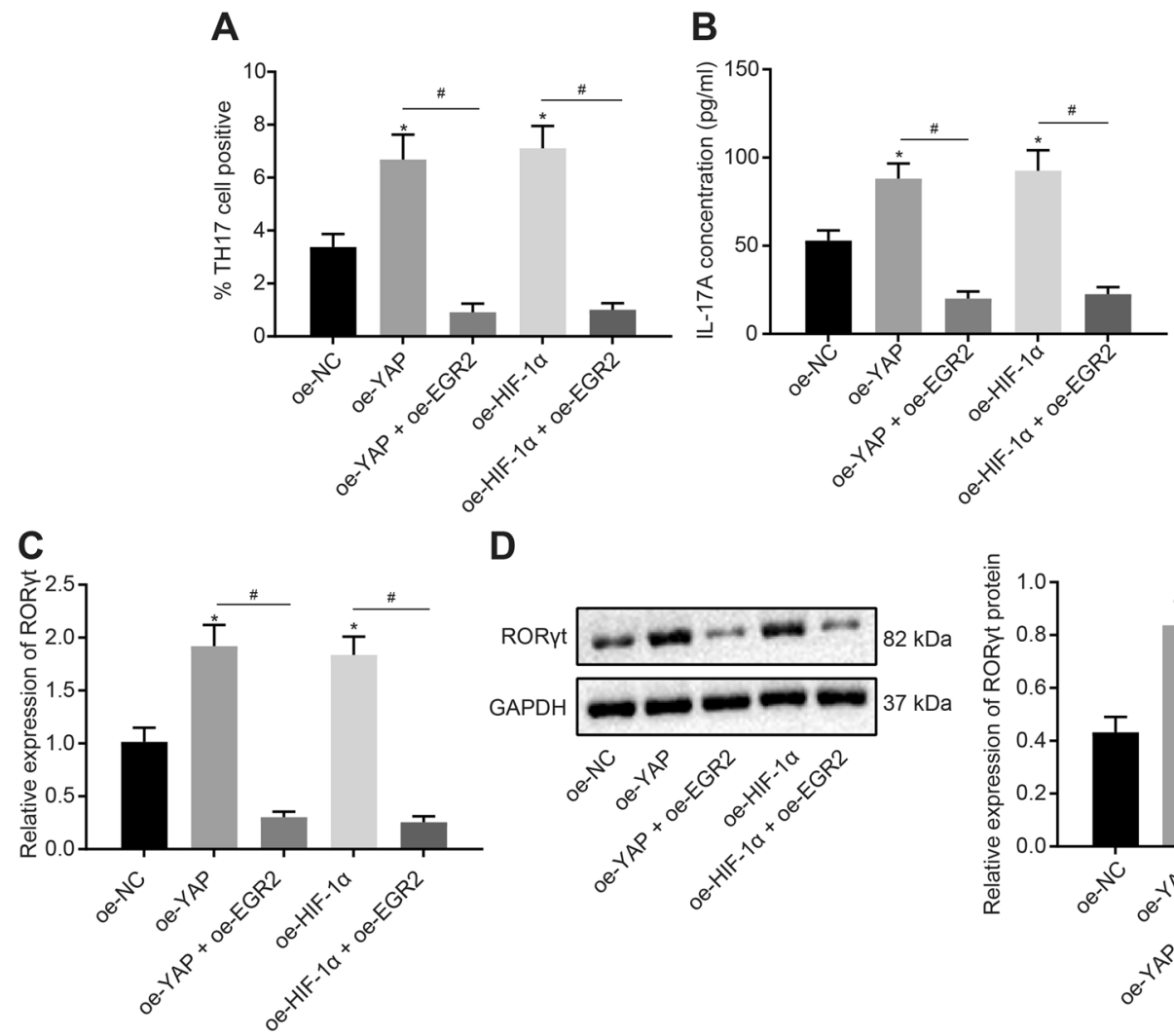

D

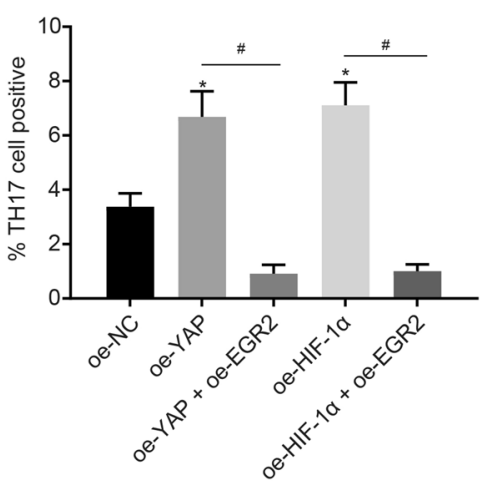

Fig. 6 Overexpression of EGR2 inhibits Th17 cell differentiation induced by the YAP/HIF-1a/miR-182 signaling. a Th17 cell proportion after different treatments determined by flow cytometry; $\mathbf{b}$ the level of IL-17A in cell supernatant after different treatments measured by ELISA; $\mathbf{c}$ RORYt mRNA expression in cells after different treatments determined by RT-qPCR; $\mathbf{d}$ Western blot analysis of RORyt protein in cells after different treatments. Comparisons between multiple groups were conducted by one-way ANOVA with Tukey's post hoc test; the experiments were repeated 3 times independently; * $p<0.05$, compared with the cells treated with oe-NC; $\# p<0.05$, compared with the cells treated with oe-YAO or oe-HIF-1a

differentiation, consequently exacerbating asthma and lipid metabolism dysfunction via miR-182-mediated EGR2 inhibition, thus providing a theoretical basis that could direct therapeutic strategies against asthma and lipid metabolic disorder.

One of the important findings of the current study is that the factors YAP1 and HIF-1 $\alpha$ were both increased in pediatric asthma. A previous study corroborated that YAP was upregulated in the bronchial airway smooth muscles of chronic asthma mouse models [28]. YAP can also bind to HIF- $1 \alpha$ in the nucleus and sustain HIF- $1 \alpha$ protein stability in conditions of hypoxic stress in hepatocellular carcinoma cells [12]. Silencing YAP has been documented to markedly downregulate the protein expression of HIF-1 $\alpha$, while inhibition of the YAP/ HIF- $1 \alpha$ signaling aids in the prevention of angiogenesis of liver sinusoidal endothelial cells [29]. These previous findings in conjunction with our results support a positive relationship exists between YAP and HIF-1 $\alpha$. Other studies have illustrated that deficiency of HIF- $1 \alpha$ can reduce eosinophil infiltration, goblet cell hyperplasia, and levels of cytokines IL-4, IL-5, and IL-13 in the lungs of OVA-induced asthma models [13], further highlighting the importance of the elevated levels of HIF-1 $\alpha$ in asthma. Moreover, HIF- $1 \alpha$ has been reported to facilitate the differentiation of Th17 cells [30], and may serve as an important signaling molecule for the induction of asthma by promoting Th17 cells $[16,17]$. HIF- $1 \alpha$ can also cause asthma by means of airway smooth muscle remodeling $[31,32]$, which is in line with our findings. Notably, YAP is known to function as an amplifier of the regulatory $\mathrm{T}$ cells Treg-reinforcing pathway, holding significant potential as an anticancer immunotherapeutic target [10]. In addition to that, loss of YAP in T cells is known to result in enhanced T-cell activation, differentiation, and function, which translates in vivo to an improved ability for $\mathrm{T}$ cells to infiltrate and repress the development of tumors [33]. These aforementioned findings and results indicate the promoting role of YAP/HIF- $1 \alpha$ in enhancing differentiation of Th17 cells. 
Lipid metabolism dysfunction is another potential problem faced by patients plagued by asthma. Accordingly, reductions in good cholesterol levels of HDL-C have been found in asthmatic children [22]. In addition to reduced HDL-C, abnormally high LDL-C and triglycerides are also a common occurrence in pediatric asthma patients [34]. More importantly, the reduction in HDL-C levels has been linked to an increased number of Th17 cells [21]. The significance of this phenomenon is reflected by the fact that Th17 cells are associated with the production of IL-17, a highly pro-inflammatory cytokine [35]. Th17 cells also produce other proinflammatory cytokines such as IL- 6 and tumor necrosis factor- $\alpha$, which play trivial roles in the inflammatory cascade stimulated in the state of asthma [36]. In particular, Th17 cells are understood to increase immune hyperresponsiveness in an enhanced inflammatory state during asthma [37]. Ni et al, have demonstrated that loss of YAP results in dysfunctional Treg cells that fail to inhibit anti-tumor immunity or elicit tumor growth in mice percentage of Treg cells; specifically, YAP-1 overexpression in hepatocellular carcinoma $\mathrm{T}$ cells induces immunosuppression by promoting Treg cell differentiation [38]. This finding is consistent with the results from this work. It indicates that the role of YAP in Th17 generation could be bidirectional, relevant to both overexpression or deletion.

Another focus of the current study was miRNAs, small non-coding RNA molecules that can modulate gene expression posttranscriptionally by interacting with the $3^{\prime}$ UTR of specific target mRNAs [39]. Herein, we identified that miR-182 could bind to the $3^{\prime}$ UTR of EGR2 and inhibit its expression. Accumulating evidence has shown that EGR2 exerts an inhibitory role on Th17 cell differentiation by negatively regulating Batf [20]. A previous study had found upregulated expression of inflammatory transcription factors, such as RORyt and Bhlhe40, in EGR2/3 deficient $T$ cells under tolerogenic conditions [40]. Furthermore, EGR2 has shown the potential to retard the development of chronic rhinosinusitis induced

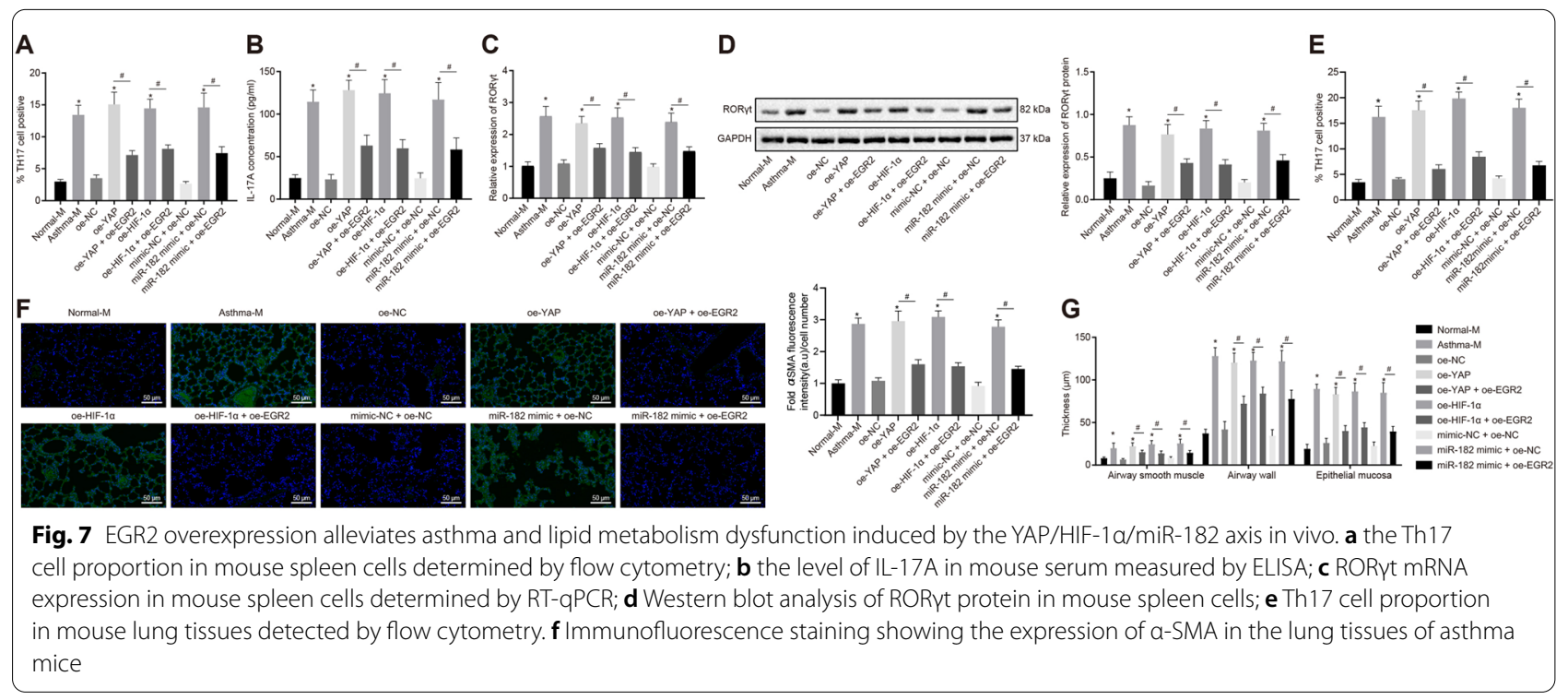

[10]. Additionally, YAP deficiency in T cells enhances T-cell activation, differentiation, and function, as well as improving T-cell responses in cancer [33]. By contrast, the results obtained from the present study revealed that YAP could potentiate Th17 cell differentiation both in PBMCs and mice with asthma. This discrepancy may be due to the differences attributable to the laboratory environment, study subjects, and the detection methods used. However, a previous study found significant overexpression and activation of YAP-1 in PBMCs collected from a total of 152 hepatocellular carcinoma cases, and showed that YAP-1 shares a positive correlation to the by miR-150-5p in dendritic cells [41]. On the other hand, previous studies have demonstrated that miR-182 may promote asthma by stimulating inflammation [42]. Our results also support the notion that miRNAs, like miR182, miR-30 [43] and miR-221 [44], could be potential candidate targets for the development of new treatments of asthma [45]. In line with our findings, miR-182 was found significantly upregulated in Th17 differentiation [15]. Therefore, we reasoned that miR-182 led to Th17 cell differentiation in pediatric asthma by targeting EGR2 as miR-182 is found capable of elevating the HIF- $1 \alpha$ expression, thus promoting breast cancer cell proliferation and 
invasion [46], and such evidence implied positive correlation between miR-182 and HIF-1 $\alpha$. HIF-1 $\alpha$-deficient mice are shown to exhibit elevated metabolic rate, hyperventilation, and improved glucose and lipid homeostasis [47]. Based on these results we validated the hypothesis that amplified EGR2 eliminated Th17 cell differentiation, asthma, and lipid metabolism dysfunction driven by $\mathrm{YAP} / \mathrm{HIF}-1 \alpha / \mathrm{miR}-182$ signaling.

\section{Conclusions}

In conclusion, the findings in the current study demonstrated that YAP/HIF-1 $\alpha$ enhanced Th17 cell differentiation, consequently exacerbating asthma and lipid metabolism dysfunction via miR-182-mediated EGR2 inhibition (Fig. 8). Thus, the YAP/HIF-1 $\alpha /$ miR-182/EGR2 signaling may serve as a novel biomarker for asthma diagnosis and prognosis. However, there are a few notable limitations to our study. First, only OVA was used to stimulate asthma in the mice in this study. While human asthma can stem from different causes and exhibit variable forms and severity, animal models can be used to mimic one of more features of the human variation of the disease $[48,49]$ and therefore, in future, different animal models should be used to confirm the results uncovered in our study to prevent over-generalization and extrapolation to human situations. Also, in the mouse models with adenovirus-driven expression of YAP, HIF- $1 \alpha$, miR182 and EGR2, it was hard to distinguish the effect of the expressed genes in different cell types, therefore, the epithelial cells from trachea to respiratory bronchioles, the alveolar ducks, and alveoli squamous type I alveolar cells, and type II alveolar cells should also be infected in future investigation. In general, further studies with different cell lines, different disease models, and larger cohorts are essential to validate the findings of the current study and validate the translational potential of the YAP/HIF- $1 \alpha /$ miR-182/EGR2 signaling axis in asthma.

\section{Material and methods}

\section{Ethics statement}

The current study was approved by the Ethics Committee of The First Affiliated Hospital of Nanchang University (approval number: 201908029) and performed in strict accordance with the Declaration of Helsinki. Signed informed consent was obtained from all participants prior to their participation in the study. Animal experiments were strictly designed and performed in compliance with the Guide for the Care and Use of Laboratory animals published by the US National Institutes of Health, and extensive efforts were made to minimize the suffering of the animals used in the study.

\section{Study Subjects}

A total of 29 pediatric patients with asthma (aged $<18$ years old; 14 males \& 15 females) and 22 healthy volunteers from the First Affiliated Hospital of Nanchang University from June 2018 to December 2018 were enrolled in the current study, and PBMCs were collected in order to extract RNA and protein content.

\section{OVA-Induced Murine Asthma Model}

A total of $120 \mathrm{BALB} / \mathrm{C}$ mice (aged 6-8 weeks; weighing $16-20 \mathrm{~g}$ ) were purchased from the Experimental Animal Center of Nanchang University, amongst which 12 mice were used as normal control, 96 mice were subjected to adenovirus infection, while the remaining 12 mice were used for asthma model establishment. The asthma models were constructed according to a previously reported method [50], with some additional adjustments. Firstly, the mice were subjected to intraperitoneal injections with $0.2 \mathrm{~mL}$ of OVA/aluminum hydroxide on days 0,7 , and 14. Thereafter, starting from day 21 , the mice were exposed to $1 \%$ OVA inhalation $(30 \mathrm{~mL})$ for $30 \mathrm{~min}$ once per day, for a total of 7 days. At every time point prior to OVA inhalation, each mouse was intraperitoneally injected with $0.2 \mathrm{~mL}$ of normal saline. Normal control mice were subjected to matching procedures, with the exception of $30 \mathrm{~min}$ of OVA inhalation treatment. For the mice subjected to adenovirus infection, 1 week before administration of OVA and normal saline, the mice were intratracheally injected with phosphate buffer saline (PBS) or adenovirus harboring overexpression plasmids for YAP, EGR2, and HIF-1 $\alpha$, miR-182 mimic, or the negative control $(\mathrm{NC})$ plasmids $\left(20 \mu \mathrm{L}, 5 \times 10^{10} \mathrm{pfu} / \mathrm{mL}\right)$ (Fubio Biological Technology Co., Ltd., Shanghai, China) [51]. After model establishment, the mice were anaesthetized with intraperitoneal injections of 3\% sodium pentobarbital (Sigma-Aldrich, St. Louis, MO, USA).

\section{Cell isolation and infection}

HEK293T cells purchased from the American Type Culture Collection (Manassas, VA, USA) were cultured in high-glucose Dulbecco's modified Eagle medium (DMEM) (10569044, Gibco, Langley, OK, USA) supplemented with 10\% fetal bovine serum (FBS) (10099141, Gibco), $100 \mathrm{U} / \mathrm{mL}$ of penicillin, and $100 \mathrm{U} / \mathrm{mL}$ of streptomycin (15070063, Gibco).

Spleen specimens from normal BALB/c mice were collected, cut into pieces using surgical scissors and detached using Collagenase D for $30 \mathrm{~min}$ at room temperature, followed with treatment using $5 \mathrm{mM}$ of ethylene diamine tetra-acetic acid for $5 \mathrm{~min}$ to harvest spleen cells. Next, naive $\mathrm{CD} 4^{+} \mathrm{T}$ cells were isolated from the harvested spleen cells with the help of $\mathrm{CD} 4{ }^{+} \mathrm{CD}_{2} \mathrm{~L}^{+} \mathrm{T}$ 


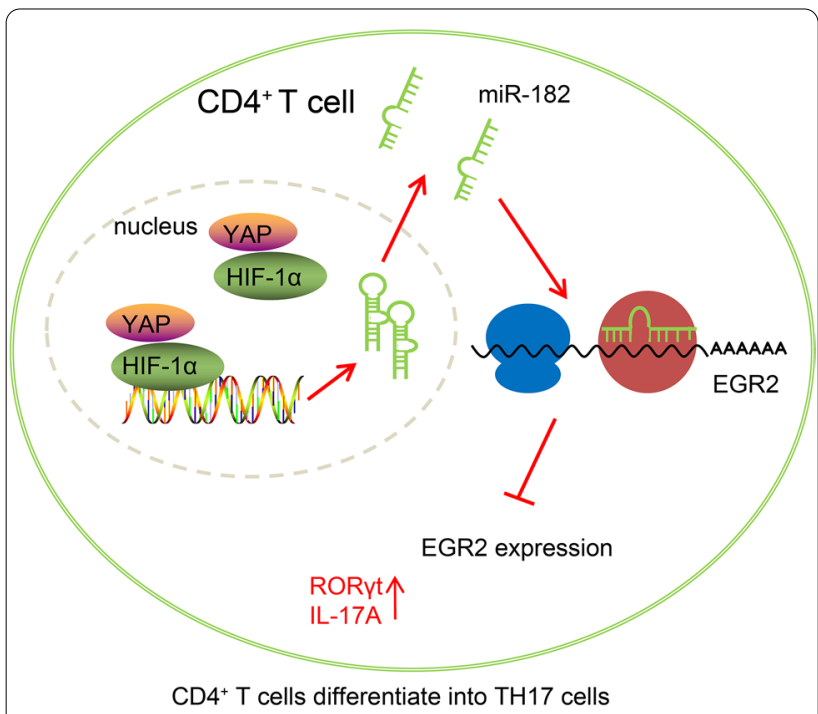

Fig. 8 A schematic diagram illustrating the role of the YAP/HIF-1a/ miR-182/EGR2 axis in asthma. The overexpression of YAP and HIF-1a promoted the expression of miR-182, thereby inhibiting EGR2 expression, increasing the expression of RORyt and IL-17A, and prompting the differentiation of $\mathrm{CD}^{+}{ }^{+} \mathrm{T}$ cells into Th 17 cells, which ultimately aggravated asthma and lipid metabolism dysfunction cell isolation kits (130-106-643, Miltenyi Biotech, Germany), with a purity of $95 \%$.

Subsequently, the obtained $\mathrm{CD}^{+} \mathrm{T}$ cells were seeded in a 6-well plate with Roswell Park Memorial Institute (RPMI)-1640 medium containing 10\% FBS at a density of $5 \times 10^{5}$ cells/well. Upon reaching $50-70 \%$ confluence, cells were treated with plasmids of miR-182 mimic, miR182 inhibitor, overexpression and silencing adenoviruses carrying YAP, HIF-1 $\alpha$, and EGR2, as well as their corresponding NCs (mimic-NC, inhibitor-NC, oe- NC, and sh-NC) purchased from Sangon Biotech (Shanghai, China) for $24 \mathrm{~h}$, followed by 1 -week treatment with puromycin. After that, RT-qPCR and Western blot analysis were performed to measure the efficiency of cell infection. The sequences for mimic and inhibitor are listed in Table 2.

In order to differentiate the $\mathrm{CD}^{+} \mathrm{T}$ cells into Th17 cells, the infected $\mathrm{CD}_{4}{ }^{+} \mathrm{T}$ cells were incubated in RPMI1640 medium containing 10\% FBS, $10 \mathrm{mM} \mathrm{N}$-2-hydroxyethyl-piperazine-N'-2-ethanesulfonic acid, $100 \mathrm{U} /$ $\mathrm{mL}$ of penicillin, $100 \mathrm{mg} / \mathrm{mL}$ of streptomycin, $50 \mathrm{mg} /$ $\mathrm{mL}$ of gentamicin, $1 \mathrm{mM}$ sodium pyruvate, $55 \mathrm{mM}$ 2-ME, $1 \mathrm{mM}$ non-essential amino acids, and $2 \mathrm{mM}$ L-glutamine at $37{ }^{\circ} \mathrm{C}$ with $5 \% \mathrm{CO}_{2}$ in air for a duration of $72 \mathrm{~h}$. RPMI medium was prepared with the addition of anti-CD3 $(10 \mu \mathrm{g} / \mathrm{mL})$ (MAB4841), anti-CD28 $(1 \mu \mathrm{g} /$ $\mathrm{mL}$ ) (MAB4832), transforming growth factor $\beta$ (TGFß) $(2 \mathrm{ng} / \mathrm{mL})(7666-\mathrm{MB}), \mathrm{IL}-6(10 \mathrm{ng} / \mathrm{mL})(406-\mathrm{ML})$,
anti-IL-4 (10 $\mu \mathrm{g} / \mathrm{mL})$ (MAB404), and anti-interferon- $\gamma$ $(\mathrm{IFN}-\gamma)(10 \mu \mathrm{g} / \mathrm{mL})(485-\mathrm{MI})$, which were all purchased from R\&D Systems (Minneapolis, MV, USA).

\section{Hematoxylin-eosin staining}

Lung tissue sections were fixed with $4 \%$ paraformaldehyde at room temperature, and then subjected to hematoxylin-eosin staining (hematoxylin for $60 \mathrm{~s}$ and eosin for $3 \mathrm{~min}$ ) for airway lesion observation. Each section was observed under an optical microscope (BX63, Tokyo, Japan) in a double-blinded manner, followed by measuring of the thickness of the airway smooth muscle $(\mu \mathrm{m})$, airway wall $(\mu \mathrm{m})$, and airway epithelium mucosa $(\mu \mathrm{m})$.

\section{Preparation of single-cell suspension from lung tissue} Mice lungs were perfused with PBS and finely minced before treatment with digestion buffer containing Collagenase D (Roche) and $\mathrm{MgCl}_{2}$, and $0.15 \mathrm{mg} / \mathrm{mL}$ DNase I (Sigma) in DMEM (HyClone). Lung perfusion is a widely accepted method for the preparation of lung immune cells [52-54], including cells trapped in capillaries [55]. Lung perfusion was applied in our study to include both immune cells from lung parenchyma and also capillaries since tissue capillary-associated immune populations are pivotal to immune responses [56-58]. Lungs were digested for $20-25 \mathrm{~min}$ at $37^{\circ} \mathrm{C}$ at $200 \mathrm{rpm}$ and then passed through $100 \mathrm{~mm}$ cell strainer to prepare a single-cell suspension.

\section{Detection of Th17 cell proportion}

Spleen tissues were isolated from C57BL/6 mice (age: 6-8 weeks), placed in a sterile culture dish, added with $5 \mathrm{~mL}$ autoMACS Running Buffer (Miltenyi Biotec) and rapidly ground as reported in a previous study [59]. A single-cell suspension was obtained by filtering through a $40 \mu \mathrm{m}$ nylon membrane. Peripheral blood monocytes were isolated and collected from healthy humans. $\mathrm{CD}^{+} \mathrm{CD}^{+} 5^{-}$cell population was harvested through autoMACS Pro Cell Separator. Then, $1 \times 10^{5}$ $\mathrm{CD} 4{ }^{+} \mathrm{CD} 25^{-}$cells were added into a 96 -well plate, and infected with adenovirus for $24 \mathrm{~h}$. Each well was coated with CD3 antigen, washed with PBS 3 times and supplemented with $100 \mu \mathrm{L}$ iTh17 mixture $(1.5 \mu \mathrm{g} / \mathrm{mL}$ antiCD28, $20 \mathrm{ng} / \mathrm{mL}$ IL- 6 and $5 \mathrm{ng} / \mathrm{mL}$ TGF- $\beta$ ). The control mixture was activated and added as NC. After $72 \mathrm{~h}$, Th17 cell differentiation was detected using a CytoFLEX flow cytometer (Beckman, Brea, CA, USA).

\section{RNA isolation and analysis}

For measuring miRNA and mRNA expression, the total RNA was extracted from tissues and cells using the TRIzol reagent (6096020, Thermo Fisher Scientific, 
Waltham, MA, USA). After that, the obtained RNA was reverse transcribed into complementary DNA (cDNA) using TaqMan ${ }^{\mathrm{TM}}$ MicroRNA Reverse Transcription Kit (4366596, Thermo Fisher Scientific) for miR182, and High-Capacity cDNA Reverse Transcription Kit (4368813, Thermo Fisher Scientific) for mRNAs. Then, RT-qPCR was performed using a RT-qPCR kit (11732020, Thermo Fisher Scientific) on a Real-Time PCR system (CFX96, Bio-Rad, Hercules, CA, USA). Finally, the expression levels of miRNA and mRNA were calculated using the $2^{-\Delta \Delta \mathrm{Ct}}$ method, the internal reference set as U6 for miR-182 and glyceraldehyde3 -phosphate dehydrogenase (GAPDH) for YAP, HIF-1 $\alpha$, and EGR2. The primer sequences were designed by Shanghai Sangon Biotech (Shanghai, China) and are presented in Table 3.

\section{Protein isolation and analysis}

For assessment of protein expression, the total protein content was extracted from tissues and cells with a protein extraction kit (78501, Thermo Fisher Scientific), followed by determination of the protein concentration using a bicinchoninic acid kit (23229, Thermo Fisher Scientific). The protein was then electroblotted onto polyvinylidene difluoride membranes (1620177, Bio-Rad), which was blocked with $5 \%$ skim milk or $5 \%$ bovine serum albumin (BSA). The membranes were subsequently probed with the following primary antibodies at $4{ }^{\circ} \mathrm{C}$ overnight: GAPDH (internal control, ab181602, dilution ratio of 1: 5000, Abcam, Cambridge, UK), YAP (ab205270, dilution ratio of 1: 1000, Abcam), HIF- $1 \alpha$ (ab2185, dilution ratio of 1: 1000, Abcam), EGR2 (ab108399, dilution ratio of 1: 1000, Abcam), and RORyt (MAB6109, dilution ratio of 1: 1000, R\&D Systems). The following day, the membranes were re-probed with horseradish peroxidase-labeled secondary goat antirabbit IgG (ab6721, dilution ratio of 1: 5000, Abcam) or rabbit anti-mouse IgG (ab6728, dilution ratio of 1: 5000, Abcam) for $1 \mathrm{~h}$ at room temperature. After that, the membranes were visualized using an enhanced chemiluminescence reagent $(1,705,062$, Bio-Rad) and analyzed using Image Quant LAS 4000C (GE Company, USA). The protein expression was quantified using ImageJ $1.48 \mathrm{u}$ software (National Institutes of Health) and expressed as the gray value of the protein to be tested to that of GAPDH.

\section{Dual luciferase reporter gene assay}

The possible binding site between miR-182 and the 3'UTR of EGR2 was predicted using a web based tool (http://starbase.sysu.edu.cn/). Next, synthesized fragments of EGR2-wt (5'-gaggccetctgtccgagctgccaac-3'),
Table 2 Primer sequences for reverse transcription quantitative polymerase chain reaction

\begin{tabular}{|c|c|}
\hline Primer & Sequence $\left(5^{\prime}-3^{\prime}\right)$ \\
\hline hYAP & $\begin{array}{l}\text { F:TAGCCCTGCGTAGCCAGTTA } \\
\text { R:TCATGCTTAGTCCACTGTCTGT }\end{array}$ \\
\hline \multirow[t]{2}{*}{ mYAP } & F: CCCTCACCCTCCCTGAAATCTACAA \\
\hline & R: AGCATTCCACAGGTCCAAGGCAGA \\
\hline $\begin{array}{l}\text { hHIF-1a } \\
\text { mHIF-1a } \\
\text { hmiR-182 } \\
\text { mmiR-182 } \\
\text { hEGR2 } \\
\text { mEGR2 } \\
\text { hRORyt } \\
\text { mRORyt } \\
\text { hGAPDH } \\
\text { mGAPDH } \\
\text { hU6 } \\
\text { mU6 }\end{array}$ & $\begin{array}{l}\text { F: GCTGGCCCCAGCCGCTGGAG } \\
\text { R: GAGTGCAGGGTCAGCACTAC } \\
\text { F: AGCTTCTGTTATGAGGCTCACC } \\
\text { R: TGACTTGATGTTCATCGTCCTC } \\
\text { F: ACACTCCAGCTGGGTTTGGCAATGGTAGAACT } \\
\text { R: TGGTGTCGTGGAGTCG } \\
\text { F: TGCGGTTTGGCAATGGTAGAAC } \\
\text { R: TGGTGTCGTGGAGTCG } \\
\text { F: CTTTGACCAGATGAACGGAG } \\
\text { R: CCCATGTAAGTGAAGGTCTG } \\
\text { F: CTTCAGCCGAAGTGACCACC } \\
\text { R: GCTCTTCCGTTCCTTCTGCC } \\
\text { F: GCTGTGATCTTGCCCAGAACC } \\
\text { R: TGCCCATCATCATTGCTGTTAATCC } \\
\text { F: TGCAAGACTCATCGACAAGG } \\
\text { R: AGGGGATTCAACATCAGTGC } \\
\text { F: GGAGCGAGATCCCTCCAAAAT } \\
\text { R: GGCTGTTGTCATACTTCTCAGG } \\
\text { F: AGGTCGGTGTGAACGGATTTG } \\
\text { R:TGTAGACCATGTAGTTGAGGTCA } \\
\text { F: GTAATACGACTCACTATAGGGAGAAGAG } \\
\text { R: CGCGCCTGCAGGTCGAC } \\
\text { F: GCTTCGGCAGCACATATACTAAAAT } \\
\text { R: CGCTTCACGAATTTGCGTGTCAT }\end{array}$ \\
\hline hmiR-183 & $\begin{array}{l}\text { F: GAGGATCCCCGGGTACCAAGGGAGTGGGCAGGCTA } \\
\text { R: ATAAGCTTGATATCGTCCCTGCACCCTTGGAAGCA }\end{array}$ \\
\hline \multirow[t]{2}{*}{ mmiR-183 } & F: CGCGCTAT GGCACTGGTAG \\
\hline & R; GTGCAGGGTCC GAGGT \\
\hline \multirow[t]{2}{*}{ hmiR-96 } & F: TTC TTC TAG AGG AAG CCC TAA TCC \\
\hline & R: TCC CTT CTA GAA AGA TCT ACT CCC C \\
\hline mmiR-96 & $\begin{array}{l}\text { F: GCCC GCTTTGGCACTAGCACATT } \\
\text { R: CAGTGCAGGGTCC GAGGT }\end{array}$ \\
\hline hEGR2 & $\begin{array}{l}\text { F: CAGGTGTCCACTCCCAGGTCCAAG } \\
\text { R: GGCAACTAGAAGGCACAGTCGAGG }\end{array}$ \\
\hline
\end{tabular}

YAP Yes-associated protein, HIF-1a hypoxia-inducible factor $1 a$, miR microRNA, $E G R 2$ early growth response 2, $F$ forward, $R$ reverse, GAPDH glyceraldehyde-3phosphate dehydrogenase, $h$ before gene name, Homo sapiens (human); $m$ before gene name, Mus musculus (house mouse)

EGR2-mut (5'-gaggccctctgtccgagcacggttc-3'), miR182-wt, and miR-182-mut (Ribo, Guangzhou, Guangdong, China) were introduced into a pGL3 vector (E1751, PromegaCorp., Madison, WI, USA) using T4 DNA ligase (M0204S, New England Biolabs Inc., MA, USA) and restriction endonuclease. These luciferase reporter plasmids were co-transfected with oe-NC, oe-HIF- $1 \alpha$, mimic-NC, and miR-182 mimic into HEK293T cells, respectively, and cultured for a duration of $48 \mathrm{~h}$. The luciferase activity was measured using a Dual-Luciferase $^{\circledR}$ Reporter Assay System kit (E1910, Promega) on a GLomax 20/20 Luminometer fluorescence detector (E5311, Promega). The luminescent signal reflecting 
the activation of the target reporter gene was computed using the ratio of the firefly relative light units (RLU) to the Renilla RLU. All vectors were constructed by Shanghai Sangon Biotechnology Co. Ltd. (Shanghai, China).

\section{HDL-C determination}

The HDL-C content was determined according to the manual of the HDL-C detection kit (ab65390, Abcam).

\section{ELISA}

Naive CD4 $+\mathrm{T}$ cells were infected with adenoviruses and differentiated in Th17 cell differentiation medium for $72 \mathrm{~h}$. The levels of IL-17A in Th17 cells of mice were measured using an IL-17A ELISA kit (M17AF0, R\&D Systems) according to the manufacturer's instructions. The serum levels of IL-17A in human were measured according to the instructions provided in the ELISA kit (D1700, R\&D Systems).

\section{ChIP assay}

The ChIP assay was conducted using a EZ-Magna ChIP kit (EMD Millipore, USA) according to standard instructions. The $\mathrm{CD} 44^{+} \mathrm{CD} 25^{-}$cell population was activated to produce Th17 cells by iTh17mixture, followed by flow cytometric sorting to screen positive Th17 cells. In brief, $1 \times 10^{6}$ Th17 cells were fixed with $1 \%$ paraformaldehyde and cross-linked with glycine for $10 \mathrm{~min}$ to produce DNA-protein cross-linking. The cells were then subjected to sonication to shear the DNA into 200-300 bp fragments. The supernatant was then collected and incubated with protein-A coated magnetic beads, followed by the addition of IgG (ab172730, dilution ratio of 1: 100, Abcam) or antibody against HIF- $1 \alpha$ (ab2185, dilution ratio of 1: 20, Abcam). The protein-DNA complexes immobilized by magnetic beads were washed and de-crosslinked. Finally, the miR-182 promoter region in the complexes was determined by RT-qPCR (miR-182 ChIP primer: Forward: 5'-GAGTGTCCAGGGTTCGTC TG-3', Reverse: 5'-GGTACACTTCTTTGCCCCCA-3').

Table 3 The degree of genes in PPI by GeneMANIA web-tool

\begin{tabular}{ll}
\hline Gene & Degree \\
\hline NOTCH1 & 36 \\
YAP1 & 33 \\
H2AFX & 15 \\
RUNX1T1 & 15 \\
USF1 & 10
\end{tabular}

PPI protein protein interaction, YAPYes-associated protein

\section{Immunofluorescence staining}

After rewarming for $30 \mathrm{~min}$, the frozen lung tissue Sects. $(5-7 \mu)$ of mice were fixed with $4 \%$ formaldehyde for $15 \mathrm{~min}$ at room temperature, and then treated with $0.2 \%$ Triton X-100 at $4{ }^{\circ} \mathrm{C}$ for $30 \mathrm{~min}$. Next, the sections were blocked using $1 \%$ BSA at room temperature for $60 \mathrm{~min}$, and subsequently incubated with mouse polyclonal anti- $\alpha$-SMA primary antibody (a2547, dilution ratio of 1: 400, Sigma) in stable buffer containing $1 \%$ BSA at room temperature for $60 \mathrm{~min}$. Then the tissue sections were incubated with goat anti mouse secondary antibody (A-11001, dilution ratio of 1: 1000, Thermo Fisher Scientific) at room temperature for $60 \mathrm{~min}$. Finally, the sections were incubated with 4,6-diamino-2-phenylindole (DAPI) (D523, dilution ratio of 1: 1000, DojinChemical Co., Kumamoto, Japan) for 10 min in the dark. Immunofluorescence images were collected using a laser scanning confocal microscope (A1RMP, Nikon, Tokyo, Japan). The fluorescence intensity was quantified and analyzed by Image-J software.

\section{Statistical analysis}

The Statistic Package for Social Science 21.0 statistical software (IBM Corp., Armonk, NY, USA) was used for statistical analyses. Measurement data were expressed as mean \pm standard deviation. Comparisons between two groups were performed using unpaired $t$-test, and comparisons between multiple groups were performed using one-way analysis of variance (ANOVA) with Tukey's post-hoc test. A value of $p<0.05$ was considered to be statistically significant.

\section{Abbreviations}

YAP: Yes-associated protein; miR: MicroRNA; EGR2: Early growth response 2; PBMCs: Peripheral blood mononuclear cells; miR-182: MicroRNA-182; Th17: T-helper 17; HDL-C: High-density lipoprotein cholesterol; PBS: Phosphate buffer saline; NC: Negative control; EDTA: Ethylene diamine tetra-acetic acid; RPMI: Roswell Park Memorial Institute; RT-qPCR: Reverse transcription quantitative polymerase chain reaction; NEAA: Non-essential amino acids; FITC: Fluorescein isothiocyanate; GAPDH: Glyceraldehyde-3-phosphate dehydrogenase; BSA: Bovine serum albumin; 3'-UTR: 3'-Untranslated region; RLU: Relative light units; ChIP: Chromatin Immunoprecipitation; ANOVA: Analysis of variance; DEGs: Expressed genes; PPI: Protein-protein interaction; MEM: Multi Experiment Matrix.

\section{Supplementary Information}

The online version contains supplementary material available at https://doi. org/10.1186/s13578-021-00560-1.

Additional file 1: Figure S1. YAP1 and HIF1A may be implicated in asthma. A, the volcano plot of DEGs related to asthma in peripheral blood cells of asthma patients obtained from the GSE97049 dataset. The red points indicate significantly upregulated genes, and the green points indicate significantly downregulated genes; $B$, the Venn diagram of the DEGs in peripheral blood cells of asthma patients from the GSE97049 dataset and the human transcription factors obtained from the Cistrome database; $C$, the PPI network of the 5 intersecting transcription factors in 
panel $B$ and the related genes; the larger circle at which genes are located reflects higher core degree of the gene and the smaller circle reflects lower core degree. D, the co-expression of YAP1 and HIF1A predicted by the MEM website $(p=3.76 \mathrm{e}-18)$; $E$, the target relationship between YAP1 and HIF1A predicted by the hTFtarget website.

Additional file 2: Figure S2. A mouse model of asthma was successfully developed. A, Hematoxylin-eosin staining of mouse lung tissues (400 $x$ ): $B$, diagram depicting the thickness of airway smooth muscle, airway wall, and airway epithelium mucosa of mice.

Additional file 3: Figure S3. YAP/HIF-1a is upregulated while HDL-C is downregulated in mice with asthma. A, the serum level of IL-17A in asthma mice measured by ELISA; B, RORYt mRNA expression in mouse spleen cells determined by RT-qPCR; C, Western blot analysis of RORYt protein in mouse spleen cells; $D, m R N A$ expression of YAP and HIF-1a in mouse spleen cells determined by RT-GPCR; E, Western blot analysis of YAP and HIF-1a proteins in mouse spleen cells. F, the serum level of HDL-C in asthma mice; G, miR-183/96/182 expression in mouse spleen cells determined by RT-qPCR. Comparisons between two groups were conducted using unpaired $t$ test. ${ }^{*} p<0.05$, compared with normal mice (normal-M). $n=12$. Each experiment was repeated 3 times independently.

Additional file 4: Figure S4. Efficiency of overexpression or knockdown of YAP/HIF-1a in Th17 cells. A, the mRNA expression of YAP and HIF-1a in cells detected by RT-qPCR; B, Western blot analysis of YAP and HIF-1a proteins in cells; C, Western blot analysis of HIF-1a proteins in cells after overexpressing/silencing YAP.

Additional file 5: Figure S5. Expression of miR-182 and HIF-1a in Th17 cells. A, HDL-C level in human serum. B, HDL-C level in mouse serum. C, miR-182 expression and HIF-1 a mRNA expression in cells after different treatments determined by RT-qPCR; D, Western blot analysis of HIF-1a protein in cells after different treatments.

Additional file 6: Figure S6. Efficiency of EGR2 overexpression or knockdown in Th17 cells. A, the mRNA expression of EGR2 in cells determined by RT-qPCR; B, Western blot analysis of EGR2 protein in cells; $C$, the mRNA expression of EGR2 in cells after different treatments determined by RT-qPCR; D, Western blot analysis of EGR2 protein in cells after different treatments.

Additional file 7: Figure S7. Expression of YAP, HIF-1a and EGR2 in naive CD4+T cells. A, the mRNA expression of YAP, HIF-1a and EGR2 in cells after different treatments determined by RT-qPCR; B, Western blot analysis of YAP, HIF-1 $a$ and EGR2 proteins in cells after different treatments.

Additional file 8: Figure S8. Expression of YAP, HIF-1a, miR-182 and EGR2 in mouse spleen cells. A, miR-182 expression and the mRNA expression of YAP, HIF-1a, and EGR2 in mouse spleen cells after different treatments determined by RT-qPCR; B, Western blot analysis of YAP, HIF-1a and EGR2 proteins in mouse spleen cells after different treatments.

Additional file 9: Table S1. Differentially expressed genes identified in GSE27876 dataset.

Additional file 10: Table S2. Human transcription factors retrieved from the Cistrome database.

Additional file 11: Table S3. Differentially expressed genes obtained from the GSE64913 dataset.

Additional file 12: Table S4. Downstream genes of miR-182 identified from the mirDIP database.

\section{Acknowledgements}

We acknowledge and appreciate our colleagues for their valuable efforts and comments on this paper.

\section{Authors' contributions}

Jing Zhou, Ning Zhang and Wei Zhang designed the study. Caiju Lu and Fei Xu collated the data, carried out data analyses and produced the initial draft of the manuscript. Jing Zhou and Ning Zhang contributed to drafting the manuscript. All authors have read and approved the final submitted manuscript.

\section{Funding}

This study was supported by Natural Science Foundation of Jiangxi Province (No. 20192BAB205001).

\section{Ethics Statement}

The current study was approved by the Ethics Committee of The First Affiliated Hospital of Nanchang University (approval number: 201908029) and performed in strict accordance with the Declaration of Helsinki. Signed informed consent was obtained from all participants prior to the study. Animal experiments were strictly designed and performed according to the Guide for the Care and Use of Laboratory animals published by the US National Institutes of Health, and extensive efforts were made to minimize the suffering of the animals used in the study.

\section{Consent for publication}

Consent for publication was obtained from the participants.

\section{Competing interests}

The authors declare that they have no conflicts of interest.

\section{Author details}

${ }^{1}$ Department of Respiratory Medicine, The First Affiliated Hospital of Nanchang University, No. 17, Yongwai Street, Donghu District, Nanchang 330006, People's Republic of China. ${ }^{2}$ Department of Imaging, The First Affiliated Hospital of Nanchang University, Nanchang 330006, People's Republic of China.

Received: 19 August 2020 Accepted: 18 February 2021

Published online: 12 May 2021

${ }^{\circ}$ nces

1. Dharmage SC, Perret JL, Custovic A. Epidemiology of asthma in children and adults. Front Pediatr. 2019. https://doi.org/10.3389/fped.2019.00246.

2. To T, Stanojevic S, Moores G, Gershon AS, Bateman ED, Cruz AA, et al. Global asthma prevalence in adults: findings from the cross-sectional world health survey. BMC Public Health. 2012. https://doi.org/10.1186/1471-2458-12-204.

3. Coleman SL, Shaw OM. Progress in the understanding of the pathology of allergic asthma and the potential of fruit proanthocyanidins as modulators of airway inflammation. Food Funct. 2017;8(12):4315-24.

4. Khan DA. Allergic rhinitis and asthma: epidemiology and common pathophysiology. Allergy Asthma Proc. 2014;35(5):357-61.

5. Castillo JR, Peters SP, Busse WW. Asthma exacerbations: pathogenesis, prevention, and treatment. J Allergy Clin Immunol Pract. 2017;5(4):918-27.

6. Ahmed $\mathrm{H}$, Turner $\mathrm{S}$. Severe asthma in children-a review of definitions, epidemiology, and treatment options in 2019. Pediatr Pulmonol. 2019;54(6):778-87.

7. Papadopoulos NG, Custovic A, Cabana MD, Dell SD, Deschildre A, Hedlin G, et al. Pediatric asthma: An unmet need for more effective, focused treatments. Pediatr Allergy Immunol. 2019;30(1):7-16.

8. Calvo F, Ege N, Grande-Garcia A, Hooper S, Jenkins RP, Chaudhry SI, et al. Mechanotransduction and YAP-dependent matrix remodelling is required for the generation and maintenance of cancer-associated fibroblasts. Nat Cell Biol. 2013;15(6):637-46.

9. Owens GK, Kumar MS, Wamhoff BR. Molecular regulation of vascular smooth muscle cell differentiation in development and disease. Physiol Rev. 2004:84(3):767-801.

10. Ni X, Tao J, Barbi J, Chen Q, Park BV, Li Z, et al. YAP is essential for treg-mediated suppression of antitumor immunity. Cancer Discov. 2018;8(8):1026-43.

11. Liu L, Zhai C, Pan Y, Zhu Y, Shi W, Wang J, et al. Sphingosine-1-phosphate induces airway smooth muscle cell proliferation, migration, and contraction by modulating Hippo signaling effector YAP. Am J Physiol Lung Cell Mol Physiol. 2018;315(4):L609-21.

12. Zhang $X$, Li Y, Ma Y, Yang L, Wang T, Meng X, et al. Yes-associated protein (YAP) binds to HIF-1alpha and sustains HIF-1alpha protein stability to promote hepatocellular carcinoma cell glycolysis under hypoxic stress. J Exp Clin Cancer Res. 2018;37(1):216.

13. Crotty Alexander LE, Akong-Moore K, Feldstein S, Johansson P, Nguyen A McEachern EK, et al. Myeloid cell HIF-1alpha regulates asthma airway resistance and eosinophil function. J Mol Med (Berl). 2013;91(5):637-44. 
14. LiY, Zhang D, Wang X, Yao X, Ye C, Zhang S, et al. Hypoxia-inducible miR-182 enhances HIF1alpha signaling via targeting PHD2 and FIH1 in prostate cancer. Sci Rep. 2015;5(12495).

15. Kastle M, Bartel S, Geillinger-Kastle K, Irmler M, Beckers J, Ryffel B, et al. microRNA cluster 106a-363 is involved in Thelper 17 cell differentiation. Immunology. 2017;152(3):402-13.

16. Zou XL, Chen ZG, Zhang TT, Feng DY, Li HT, Yang HL. Th17/Treg homeostasis, but not Th1/Th2 homeostasis, is implicated in exacerbation of human bronchial asthma. Ther Clin Risk Manag. 2018;14:1627-36.

17. Chesne J, Braza F, Mahay G, Brouard S, Aronica M, Magnan A. IL-17 in severe asthma. Where do we stand? Am J Respir Crit Care Med. 2014;190(10):1094-101.

18. Zeng Z, Wang L, Ma W, Zheng R, Zhang H, Zeng X, et al. Inhibiting the Notch signaling pathway suppresses Th17-associated airway hyperresponsiveness in obese asthmatic mice. Lab Invest. 2019;99(12):1784-94.

19. Du N, Kwon H, Li P, West EE, Oh J, Liao W, et al. EGR2 is critical for peripheral naive T-cell differentiation and the T-cell response to influenza. Proc Natl Acad Sci USA. 2014;111(46):16484-9.

20. Miao T, Raymond M, Bhullar P, Ghaffari E, Symonds AL, Meier UC, et al. Early growth response gene-2 controls IL-17 expression and Th17 differentiation by negatively regulating Batf. J Immunol. 2013;190(1):58-65.

21. Lopez P, Rodriguez-Carrio J, Martinez-Zapico A, Perez-Alvarez Al, Benavente $L$, Caminal-Montero L, et al. IgM anti-phosphorylcholine antibodies associate with senescent and IL-17+ T cells in SLE patients with a pro-inflammatory lipid profile. Rheumatology (Oxford). 2020;59(2):407-17.

22. Peng J, Huang Y. Meta-analysis of the association between asthma and serum levels of high-density lipoprotein cholesterol and low-density lipoprotein cholesterol. Ann Allergy Asthma Immunol. 2017;1 18(1):61-5.

23. Fu J, Zheng $M$, Zhang $X$, Zhang $Y$, Chen $Y$, Li H, et al. Fibulin-5 promotes airway smooth muscle cell proliferation and migration via modulating Hippo-YAP/ TAZ pathway. Biochem Biophys Res Commun. 2017:493(2):985-91.

24. Fodor LE, Gezsi A, Ungvari L, Semsei AF, Gal Z, Nagy A, et al. Investigation of the possible role of the Hippo/YAP1 pathway in asthma and allergy. Allergy Asthma Immunol Res. 2017;9(3):247-56.

25. Yan B, Jiang Z, Cheng L, Chen K, Zhou C, Sun L, et al. Paracrine HGF/C-MET enhances the stem cell-like potential and glycolysis of pancreatic cancer cells via activation of YAP/HIF-1alpha. Exp Cell Res. 2018;371(1):63-71.

26. Fang TC, Yashiro-Ohtani Y, Del Bianco C, Knoblock DM, Blacklow SC, Pear WS Notch directly regulates Gata3 expression during Thelper 2 cell differentiation. Immunity. 2007;27(1):100-10.

27. Mims JW. Asthma: definitions and pathophysiology. Int Forum Allergy Rhinol. 2015;5(Suppl 1):S2-6.

28. Zhou J, Xu F, Yu JJ, Zhang W. YAP is up-regulated in the bronchial airway smooth muscle of the chronic asthma mouse model. Int J Clin Exp Pathol. 2015;8(9):11132-9.

29. Zhang C, Bian M, Chen X, Jin H, Zhao S, Yang X, et al. Oroxylin A prevents angiogenesis of LSECs in liver fibrosis via inhibition of YAP/HIF-1alpha signaling. J Cell Biochem. 2018;119(2):2258-68

30. Xu L, Sun WJ, Jia AJ, Qiu LL, Xiao B, Mu L, et al. MBD2 regulates differentiation and function of Th17 cells in neutrophils- dominant asthma via HIF-1alpha. J Inflamm (Lond). 2018. https://doi.org/10.1186/s12950-018-0191-X.

31. Baek KJ, Cho JY, Rosenthal P, Alexander LEC, Nizet V, Broide DH. Hypoxia potentiates allergen induction of HIF-1alpha, chemokines, airway inflammation, TGF-beta1, and airway remodeling in a mouse model. Clin Immunol. 2013;147(1):27-37.

32. Zhao X, Yu FQ, Huang XJ, Xu BY, Li YL, Zhao XY, et al. Azithromycin influences airway remodeling in asthma via the PI3K/Akt/MTOR/HIF-1alpha/NEGF pathway. J Biol Regul Homeost Agents. 2018;32(5):1079-88.

33. Stampouloglou E, Cheng N, Federico A, Slaby E, Monti S, Szeto GL, et al. Yap suppresses T-cell function and infiltration in the tumor microenvironment. PLoS Biol. 2020;18(1):e3000591.

34. Ko SH, Jeong J, Baeg MK, Han KD, Kim HS, Yoon JS, et al. Lipid profiles in adolescents with and without asthma: Korea National Health and nutrition examination survey data. Lipids Health Dis. 2018;17(1):158.

35. Iwakura Y, Ishigame $H$. The IL-23/IL-17 axis in inflammation. J Clin Invest. 2006:116(5):1218-22

36. Al-Ramli W, Al Samri M, Hamid Q. Th-17 cell-related cytokines' potential role in the pathogenesis of severe asthma. J Asthma. 2008;45(Suppl 1):41-4.

37. Nanda A, Wasan AN. Asthma in adults. Med Clin North Am. 2020;104(1):95-108
38. Fan Y, Gao Y, Rao J, Wang K, Zhang F, Zhang C. YAP-1 promotes tregs differentiation in hepatocellular carcinoma by enhancing TGFBR2 transcription. Cell Physiol Biochem. 2017:41(3):1189-98.

39. Ivey KN, Srivastava D. microRNAs as developmental regulators. Cold Spring Harb Perspect Biol. 2015;7(7):a008144.

40. Omodho B, Miao T, Symonds A, , Singh R, Li S, Wang P. Transcription factors early growth response gene (Egr) 2 and 3 control inflammatory responses of tolerant T cells. Immun Inflamm Dis. 2018;6(2):221-33.

41. Ma Z, Shen Y, Zeng Q, Liu J, Yang L, Fu R, et al. MiR-150-5p regulates EGR2 to promote the development of chronic rhinosinusitis via the DC-Th axis. Int Immunopharmacol. 2018;54:188-97.

42. Miller CH, Smith SM, Elguindy M, Zhang T, Xiang JZ, Hu X, et al. RBP-J-Regulated miR-182 Promotes TNF-alpha-Induced Osteoclastogenesis. J Immunol. 2016;196(12):4977-86.

43. Li BB, Chen YL, Pang F. MicroRNA-30a targets ATG5 and attenuates airway fibrosis in asthma by suppressing autophagy. Inflammation. 2020;43(1):44-53.

44. Issouf M, Vargas A, Boivin R, Lavoie JP. MicroRNA-221 is overexpressed in the equine asthmatic airway smooth muscle and modulates smooth muscle cell proliferation. Am J Physiol Lung Cell Mol Physiol. 2019;317(6):L748-57.

45. Mousavi SR, Ahmadi A, Jamalkandi SA, Salimian J. Involvement of microRNAs in physiological and pathological processes in asthma. J Cell Physiol. 2019;234(12):21547-59.

46. Chiang $\mathrm{CH}$, Chu PY, Hou MF, Hung WC. MiR-182 promotes proliferation and invasion and elevates the HIF-1alpha-VEGF-A axis in breast cancer cells by targeting FBXW7. Am J Cancer Res. 2016;6(8):1785-98.

47. Zhang N, Fu Z, Linke S, Chicher J, Gorman JJ, Visk D, et al. The asparaginyl hydroxylase factor inhibiting HIF-1alpha is an essential regulator of metabolism. Cell Metab. 2010;11(5):364-78.

48. Daubeuf F, Frossard N. Eosinophils and the ovalbumin mouse model of asthma. Methods Mol Biol. 2014;1178:283-93.

49. Maltby S, Tay HL, Yang M, Foster PS. Mouse models of severe asthma: Understanding the mechanisms of steroid resistance, tissue remodelling and disease exacerbation. Respirology. 2017;22(5):874-85.

50. Zhang $Y, X u B$, Luan B, Zhang $Y$, Wang X, Xiong X, et al. Tumor-derived MDSCs inhibit airway remodeling in asthmatic mice through regulating $\mathrm{LL}-10$ and IL-12. Am J Transl Res. 2019;11(7):4192-202.

51. Zhou Y, Do DC, Ishmael FT, Squadrito ML, Tang HM, Tang HL, et al. Mannose receptor modulates macrophage polarization and allergic inflammation through miR-511-3p. J Allergy Clin Immunol. 2018;141(1):350-64 e8.

52. Califano D, Cho JJ, Uddin MN, Lorentsen KJ, Yang Q, Bhandoola A, et al. Transcription factor Bcl11 b controls identity and function of mature type 2 innate lymphoid cells. Immunity. 2015;43(2):354-68.

53. Duerr CU, McCarthy CD, Mindt BC, Rubio M, Meli AP, Pothlichet J, et al. Type I interferon restricts type 2 immunopathology through the regulation of group 2 innate lymphoid cells. Nat Immunol. 2016;17(1):65-75.

54. von Moltke J, O'Leary CE, Barrett NA, Kanaoka Y, Austen KF, Locksley RM. Leukotrienes provide an NFAT-dependent signal that synergizes with IL-33 to activate ILC2s. J Exp Med. 2017;214(1):27-37.

55. Anderson KG, Sung H, Skon CN, Lefrancois L, Deisinger A, Vezys V, et al. Cutting edge: intravascular staining redefines lung CD8 T cell responses. J Immunol. 2012;189(6):2702-6.

56. Rodero MP, Poupel L, Loyher PL, Hamon P, Licata F, Pessel C, et al. Immune surveillance of the lung by migrating tissue monocytes. Elife. 2015;4:e07847.

57. Carlin LM, Stamatiades EG, Auffray C, Hanna RN, Glover L, Vizcay-Barrena G, et al. Nr4a1-dependent Ly6C(low) monocytes monitor endothelial cells and orchestrate their disposal. Cell. 2013;153(2):362-75.

58. Lee WY, Moriarty TJ, Wong $\mathrm{CH}$, Zhou H, Strieter RM, van Rooijen N, et al. An intravascular immune response to Borrelia burgdorferi involves Kupffer cells and iNKT cells. Nat Immunol. 2010;11(4):295-302.

59. Bedoya SK, Wilson TD, Collins EL, Lau K, Larkin J 3rd. Isolation and th17 differentiation of naive CD4 T lymphocytes. JVis Exp. 2013;79:e50765.

\section{Publisher's Note}

Springer Nature remains neutral with regard to jurisdictional claims in published maps and institutional affiliations. 